\title{
Dirichlet Problems with an Indefinite and Unbounded Potential and Concave-Convex Nonlinearities
}

\author{
Leszek Gasiński ${ }^{1}$ and Nikolaos S. Papageorgiou ${ }^{2}$ \\ ${ }^{1}$ Faculty of Mathematics and Computer Science, Jagiellonian University, ulica Łojasiewicza 6, \\ 30-348 Kraków, Poland \\ ${ }^{2}$ Department of Mathematics, National Technical University, Zografou Campus, 15780 Athens, Greece
}

Correspondence should be addressed to Leszek Gasiński, leszek.gasinski@ii.uj.edu.pl

Received 5 March 2012; Accepted 18 April 2012

Academic Editor: Yuriy Rogovchenko

Copyright (c) 2012 L. Gasiński and N. S. Papageorgiou. This is an open access article distributed under the Creative Commons Attribution License, which permits unrestricted use, distribution, and reproduction in any medium, provided the original work is properly cited.

We consider a parametric semilinear Dirichlet problem with an unbounded and indefinite potential. In the reaction we have the competing effects of a sublinear (concave) term and of a superlinear (convex) term. Using variational methods coupled with suitable truncation techniques, we prove two multiplicity theorems for small values of the parameter. Both theorems produce five nontrivial smooth solutions, and in the second theorem we provide precise sign information for all the solutions.

\section{Introduction}

Let $\Omega \in \mathbb{R}^{N}$ be a bounded domain with a $C^{2}$-boundary $\partial \Omega$. In this paper we study the following parametric nonlinear Dirichlet problem:

$$
\begin{gathered}
-\Delta u(z)+\beta(z) u(z)=\lambda g(z, u(z))+f(z, u(z)) \quad \text { in } \Omega, \\
\left.u\right|_{\partial \Omega}=0, \quad \lambda>0 .
\end{gathered}
$$

Here $\beta \in L^{s}(\Omega)$ with $s>N / 2(N \geqslant 2)$ is a potential function which may change sign (indefinite potential). Also $\lambda>0$ is a parameter, and $g, f: \Omega \times \mathbb{R} \rightarrow \mathbb{R}$ are Carathéodory functions (i.e., for all $\zeta \in \mathbb{R}$, functions $z \mapsto g(z, \zeta)$ and $z \mapsto f(z, \zeta)$ are measurable and for almost all $z \in \Omega$, functions $\zeta \mapsto g(z, \zeta)$ and $\zeta \mapsto f(z, \zeta)$ are continuous). We assume that for almost all $z \in \Omega$, the function $\zeta \mapsto g(z, \zeta)$ is strictly sublinear near $\pm \infty$, while the function $\zeta \mapsto f(z, \zeta)$ is superlinear near $\pm \infty$. So, problem $\left((P)_{\lambda}\right)$ exhibits competing nonlinearities of 
the concave-convex type. This situation was first studied with $\beta \equiv 0$ and the right hand side nonlinearity being

$$
\lambda|\zeta|^{q-2} \zeta+|\zeta|^{r-2} \zeta
$$

with

$$
1<q<2<r<2^{*}= \begin{cases}\frac{2 N}{N-2} & \text { if } N>2 \\ +\infty & \text { if } N=2\end{cases}
$$

by Ambrosetti et al. [1]. In [1], the authors focus on positive solutions and proved certain bifurcation-type phenomena as $\lambda>0$ varies. Further results in this direction can be found in the works of I'lyasov [2], Li et al. [3], Lubyshev [4], and Rădulescu and Repovš [5]. In all the aforementioned works $\beta \equiv 0$. We should also mention the recent work of Motreanu et al. [6], where the authors consider equations driven by the $p$-Laplacian, with concave term of the form $|\zeta|^{q-2} \zeta$ (where $1<q<p$ ) and a perturbation exhibiting an asymmetric behaviour at $+\infty$ and at $-\infty((p-1)$-superlinear near $+\infty$ and $(p-1)$-sublinear near $-\infty)$. They prove multiplicity results producing four nontrivial solutions with sign information.

In this work, we prove two multiplicity results for problem $\left((P)_{\lambda}\right)$ when the parameter $\lambda>0$ is small. In both results we produce five nontrivial smooth solutions, and in the second we provide precise sign information for all the solutions. For the superlinear ("convex") nonlinearity $f(z, \cdot)$, we do not employ the usual in such cases Ambrosetti-Rabinowitz condition. Instead, we use a more general condition, which incorporates in our framework also superlinear perturbations with "slow" growth near $\pm \infty$, which do not satisfy the Ambrosetti-Rabinowitz condition. We should point out that none of the works mentioned earlier provide sign information for all the solutions (in particular, none of them produced a nodal (sign changing) solution) and all use the Ambrosetti-Rabinowitz condition to express the superlinearity of the "convex" contribution in the reaction.

Our approach is variational based on the critical point theory which is combined with suitable truncation techniques. In the next section we recall the main mathematical tools we will use in the analysis of problem $\left((P)_{\curlywedge}\right)$. We also introduce the hypotheses on the terms $g$ and $f$.

\section{Mathematical Background and Hypotheses}

Let $X$ be a Banach space and let $X^{*}$ be its topological dual. By $\langle\cdot, \cdot\rangle$ we denote the duality brackets for the pair $\left(X^{*}, X\right)$. Let $\varphi \in C^{1}(X)$. We say that $\varphi$ satisfies the Cerami condition, if the following is true.

Every sequence $\left\{x_{n}\right\}_{n \geqslant 1} \subseteq X$, such that $\left\{\varphi\left(x_{n}\right)\right\}_{n \geqslant 1} \subseteq \mathbb{R}$ is bounded and

$$
\left(1+\left\|x_{n}\right\|\right) \varphi^{\prime}\left(x_{n}\right) \longrightarrow 0 \quad \text { in } X^{*},
$$

admits a strongly convergent subsequence.

This compactness-type condition is in general weaker than the usual Palais-Smale condition. However, the Cerami condition suffices to prove a deformation theorem and from 
it we derive the minimax theory for certain critical values of $\varphi \in C^{1}(X)$ (see Gasiński and Papageorgiou [7] and Motreanu and Rădulescu [8]). In particular, we can have the following theorem, known in the literature as the mountain pass theorem.

Theorem 2.1. If $X$ is a Banach space, $\varphi \in C^{1}(X)$ satisfies the Cerami condition, $x_{0}, x_{1} \in X, \rho>$ $0\left\|x_{1}-x_{0}\right\|>\rho$ :

$$
\begin{gathered}
\max \left\{\varphi\left(x_{0}\right), \varphi\left(x_{1}\right)\right\}<\inf \left\{\varphi(x):\left\|x-x_{0}\right\|=\rho\right\}=\eta_{\rho} \\
c=\inf _{\gamma \in \Gamma \in[0,1]} \varphi(\gamma(t)),
\end{gathered}
$$

where

$$
\Gamma=\left\{\gamma \in C([0,1] ; X): \gamma(0)=x_{0}, \gamma(1)=x_{1}\right\}
$$

Then $c \geqslant \eta_{\rho}$ and $c$ are a critical value of $\varphi$.

In the analysis of problem $\left((P)_{\curlywedge}\right)$, in addition to the Sobolev space $H_{0}^{1}(\Omega)$, we will also use the Banach space

$$
C_{0}^{1}(\bar{\Omega})=\left\{u \in C^{1}(\bar{\Omega}):\left.u\right|_{\partial \Omega}=0\right\}
$$

This is an ordered Banach space with positive cone:

$$
C_{+}=\left\{u \in C_{0}^{1}(\bar{\Omega}): u(z) \geqslant 0 \forall z \in \bar{\Omega}\right\} .
$$

This cone has a nonempty interior given by

$$
\text { int } C_{+}=\left\{u \in C_{+}: u(z)>0 \forall z \in \Omega, \frac{\partial u}{\partial n}(z)<0 \forall z \in \partial \Omega\right\}
$$

where $n(\cdot)$ is the outward unit normal on $\partial \Omega$.

In the proof of the second multiplicity theorem and in order to produce a nodal (sign changing) solution, we will also use critical groups. So, let us recall their definition. Let $\varphi \in$ $C^{1}(X)$ and let $c \in \mathbb{R}$. We introduce the following sets:

$$
\begin{aligned}
\varphi^{c} & =\{x \in X: \varphi(x) \leqslant c\}, \\
K_{\varphi} & =\left\{x \in X: \varphi^{\prime}(x)=0\right\} .
\end{aligned}
$$

Also, if $\left(Y_{1}, Y_{2}\right)$ is a topological pair with $Y_{2} \subseteq Y_{1} \subseteq X$ and $k \geqslant 0$ is an integer, by $H_{k}\left(Y_{1}, Y_{2}\right)$ we denote the $k$ th relative singular homology group for the pair $\left(Y_{2}, Y_{1}\right)$ with integer coefficients. The critical groups of $\varphi$ at an isolated critical point $x_{0} \in X$ of $\varphi$ with $\varphi\left(x_{0}\right)=c$ are defined by

$$
C_{k}\left(\varphi, x_{0}\right)=H_{k}\left(\varphi^{c} \cap U, \varphi^{c} \cap U \backslash\left\{x_{0}\right\}\right) \quad \forall k \geqslant 0,
$$


where $U$ is a neighbourhood of $x_{0}$, such that $K_{\varphi} \cap \varphi^{c} \cap U=\left\{x_{0}\right\}$. The excision property of singular homology implies that this definition is independent of the particular choice of the neighbourhood $U$.

Using the spectral theorem for compact self-adjoint operators, we can show that the differential operator

$$
H_{0}^{1}(\Omega) \ni u \longmapsto-\Delta u+\beta u
$$

has a sequence of distinct eigenvalues $\left\{\widehat{\lambda}_{k}\right\}_{k \geqslant 1}$, such that

$$
\hat{\jmath}_{k} \longrightarrow+\infty \text { as } k \longrightarrow+\infty
$$

The first eigenvalue is simple and admits the following variational characterization:

$$
\widehat{\jmath}_{1}=\inf \left\{\frac{\sigma(u)}{\|u\|_{2}^{2}}: u \in H_{0}^{1}(\Omega), u \neq 0\right\},
$$

where

$$
\sigma(u)=\|\nabla u\|_{2}^{2}+\int_{\Omega} \beta(z) u(z)^{2} d z \quad \forall u \in H_{0}^{1}(\Omega)
$$

Moreover, the corresponding eigenfunction $\widehat{u} \in C_{0}^{1}(\bar{\Omega})$ does not change sign, and in fact we can take $\widehat{u}(z)>0$ for all $z \in \Omega$ (see Gasiński and Papageorgiou [9]). Using (2.11) and this property of the principal eigenfunction, we can have the following lemma (see Gasiński and Papageorgiou [9, Lemma 2.1]).

Lemma 2.2. If $\eta \in L^{s}(\Omega), \eta(z) \leqslant \widehat{\lambda}_{1}$ for almost all $z \in \Omega, \eta \neq \hat{\lambda}_{1}$, then there exists $\widehat{c}>0$, such that

$$
\sigma(u)-\int_{\Omega} \eta u^{2} d z \geqslant \widehat{c}\|u\|^{2} \quad \forall u \in H_{0}^{1}(\Omega)
$$

Also, from Gasiński and Papageorgiou [9] we know that there exist $\widehat{c}_{0}, \widehat{c}_{1}>0$, such that

$$
\|u\|^{2} \leqslant \widehat{c}_{0}\left(\sigma(u)+\widehat{c}_{1}\|u\|_{2}^{2}\right) \quad \forall u \in H_{0}^{1}(\Omega),
$$

with $\|u\|=\|\nabla u\|_{2}$, the norm of the Sobolev space $H_{0}^{1}(\Omega)$.

Next we state the hypotheses on the two components $g$ and $f$ of the reaction in problem $\left((P)_{\lambda}\right)$.

Let

$$
G(z, \zeta)=\int_{0}^{\zeta} g(z, s) d s, \quad F(z, \zeta)=\int_{0}^{\zeta} f(z, s) d s .
$$


$H_{g}: g: \Omega \times \mathbb{R} \rightarrow \mathbb{R}$ is a Carathéodory function, such that $g(z, 0)=0$ for almost all $z \in \Omega$.

(i) For every $\varphi>0$, there exists a function $a_{\rho} \in L^{\infty}(\Omega)_{+}$, such that

$$
|g(z, \zeta)| \leqslant a_{\rho}(z) \text { for almost all } z \in \Omega \text {, all }|\zeta| \leqslant \rho .
$$

(ii) We have

$$
\lim _{\zeta \rightarrow \pm \infty} \frac{g(z, \zeta)}{\zeta}=0 \quad \text { uniformly for almost all } z \in \Omega
$$

(iii) There exist constants $c_{1}, c_{2}>0,1<q<\mu<2$ and $\delta_{0}>0$, such that

$$
\begin{gathered}
c_{1}|\zeta|^{q} \leqslant g(z, \zeta) \zeta \text { for almost all } z \in \Omega, \text { all } \zeta \in \mathbb{R}, \\
\mu G(z, \zeta)-g(z, \zeta) \zeta \geqslant c_{2}|\zeta|^{q} \text { for almost all } z \in \Omega \text {, all }|\zeta| \leqslant \delta_{0} .
\end{gathered}
$$

(iv) For every $\varphi>0$, we can find $\gamma_{\rho}>0$, such that for almost all $z \in \Omega$

$$
\text { the map }[-\varrho, \varrho] \ni \zeta \longmapsto g(z, \zeta)+\gamma_{\rho}|\zeta|^{q-2} \zeta \text { is nondecreasing. }
$$

Remark 2.3. Hypothesis $H_{g}($ ii) implies that for almost all $z \in \Omega$, the function $g(z, \cdot)$ is strictly sublinear near $\pm \infty$. Hence $g(z, \cdot)$ is the "concave" component in the reaction of $\left((P)_{\curlywedge}\right)$ (the terminology "concave" and "convex" nonlinearities is due to Ambrosetti [1]). Note that hypothesis $H_{g}($ iii) implies that for almost all $z \in \Omega$, the function $g(z, \cdot)$ has a similar growth near 0 that is, we have a concave term near zero. Hypothesis $H_{g}($ iv) is weaker than assuming the monotonicity of $g(z, \cdot)$ for almost all $z \in \Omega$.

$H_{f}: f: \Omega \times \mathbb{R} \rightarrow \mathbb{R}$ is a Carathéodory function, such that $f(z, 0)=0$ for almost all $z \in \Omega$.

(i) There exist $a \in L^{\infty}(\Omega)_{+}, c>0$ and $r \in\left(2,2^{*}\right)$, such that

$$
|f(z, \zeta)| \leqslant a(z)+c|\zeta|^{r-1} \quad \text { for almost all } z \in \Omega, \text { all } \zeta \in \mathbb{R}
$$

(ii) We have

$$
\lim _{\zeta \rightarrow \pm \infty} \frac{F(z, \zeta)}{\zeta^{2}}=+\infty \quad \text { uniformly for almost all } z \in \Omega .
$$

(iii) There exist functions $\eta_{0}, \hat{\eta}_{0} \in L^{\infty}(\Omega)_{+}$, such that $\eta_{0}(z) \leqslant \hat{\lambda}_{1}$ for almost all $z \in \Omega$, $\eta_{0} \neq \widehat{\lambda}_{1}$ and

$$
\hat{\eta}_{0}(z) \leqslant \liminf _{\zeta \rightarrow 0} \frac{f(z, \zeta)}{\zeta} \leqslant \limsup _{\zeta \rightarrow 0} \frac{f(z, \zeta)}{\zeta} \leqslant \eta_{0}(z)
$$

uniformly for almost all $z \in \Omega$. 
(iv) For every $\varphi>0$, we can find $\widehat{\gamma}_{\rho}>0$, such that for almost all $z \in \Omega$, we have

$$
\text { the map }[-Q, \varrho] \ni \zeta \longmapsto f(z, \zeta)+\widehat{\gamma}_{\ell} \zeta \text { is nondecreasing. }
$$

Remark 2.4. Hypothesis $H_{f}$ (ii) implies that for almost all $z \in \Omega$, the function $F(z, \cdot)$ is superquadratic near $\pm \infty$. Evidently, this is satisfied if the function $f(z, \cdot)$ is superlinear near $\pm \infty$, that is, when

$$
\lim _{\zeta \rightarrow \pm \infty} \frac{f(z, \zeta)}{\zeta}=+\infty \quad \text { uniformly for almost all } z \in \Omega
$$

So, $f(z, \zeta)$ is the "convex" component of the reaction which "competes" with the "concave" component $g(z, \zeta)$.

Note that in $H_{f}$, we did not include the Ambrosetti-Rabinowitz condition to characterize the superlinearity of $f(z, \cdot)$. We recall that the Ambrosetti-Rabinowitz condition says that there exist $\tau>2$ and $M>0$, such that

$$
\begin{gathered}
0<\tau F(z, \zeta) \leqslant f(z, \zeta) \zeta \text { uniformly for almost all } z \in \Omega, \text { all }|\zeta| \geqslant M, \\
\qquad \underset{\Omega}{\operatorname{ess} \inf F(\cdot, M)>0 .}
\end{gathered}
$$

Integrating (2.25) and using (2.26), we obtain the weaker condition

$$
c_{3}|\zeta|^{\tau} \leqslant F(z, \zeta) \quad \text { uniformly for almost all } z \in \Omega \text {, all }|\zeta| \geqslant M
$$

Therefore, for almost all $z \in \Omega$, the function

$$
\zeta \longmapsto \lambda G(z, \zeta)+F(z, \zeta)
$$

is superquadratic with at least $\tau$-growth near $\pm \infty$. Hence the Ambrosetti-Rabinowitz condition excludes superlinear perturbations with "slower" growth near $\pm \infty$. For this reason, here we employ a weaker condition. So, let

$$
\xi_{\lambda}(z, \zeta)=(\lambda g(z, \zeta)+f(z, \zeta)) \zeta-2(\lambda G(z, \zeta)+F(z, \zeta)) .
$$

We employ the following hypothesis.

$\underline{H_{0}}$ : For every $\lambda>0$, there exists a function $\vartheta_{\lambda}^{*} \in L^{1}(\Omega)_{+}$, such that

$$
\begin{aligned}
& \xi_{\lambda}(z, \zeta) \leqslant \xi_{\lambda}(z, y)+\vartheta_{\lambda}^{*}(z) \text { for almost all } z \in \Omega, \text { all } 0 \leqslant \zeta \leqslant y, \\
& \xi_{\lambda}(z, \zeta) \leqslant \xi_{\lambda}(z, y)+\vartheta_{\lambda}^{*}(z) \text { for almost all } z \in \Omega, \text { all } y \leqslant \zeta \leqslant 0 .
\end{aligned}
$$


Remark 2.5. Hypothesis $H_{0}$ is a generalized version of a condition first introduced by $\mathrm{Li}$ and Yang [10], where the reader can find other possible extensions of the Ambrosetti-Rabinowitz condition and comparisons between them. Hypothesis $H_{0}$ is a quasimonotonicity condition on $\xi_{\lambda}(z, \cdot)$, and it is satisfied if there exists $M>0$, such that for almost all $z \in \Omega$, the function

$$
\zeta \longmapsto \frac{\lambda g(z, \zeta)+f(z, \zeta)}{\zeta}
$$

is increasing on $[M,+\infty]$ and is decreasing on $[-\infty,-M]$ (see Li and Yang [10]).

Example 2.6. The following pairs of functions satisfy hypotheses $H_{g}, H_{f}$, and $H_{0}$ (for the sake of simplicity we drop the $z$-dependence):

$$
\begin{gathered}
g_{1}(\zeta)=|\zeta|^{q-2} \zeta, \quad f_{1}(\zeta)=|\zeta|^{p-2} \zeta+\eta_{0} \zeta, \\
g_{2}(\zeta)=|\zeta|^{q-2} \zeta, \quad f_{2}(\zeta)=|\zeta|^{p-2} \zeta \ln (1+|\zeta|)+\eta_{0} \zeta, \\
g_{3}(\zeta)=|\zeta|^{q-2} \zeta-|\zeta|^{\tau-2} \zeta, \quad f_{3}(\zeta)= \begin{cases}\eta_{0}\left(\zeta-|\zeta|^{r-2} \zeta\right) & \text { if }|\zeta| \leqslant 1, \\
|\zeta|^{p-2} \zeta \ln |\zeta| & \text { if }|\zeta|>1,\end{cases}
\end{gathered}
$$

with $1<q<\tau<2<p<2^{*}, 2<r, \eta_{0}<\hat{\lambda}_{1}$.

Note that $f_{2}$ and $f_{3}$ do not satisfy the Ambrosetti-Rabinowitz condition (see (2.25)$(2.26))$.

For every $u \in H_{0}^{1}(\Omega)$, we set

$$
\|u\|=\|\nabla u\|_{2}
$$

(by virtue of the Poincaré inequality). We mention that the notation $\|\cdot\|$ will be also used to denote the $\mathbb{R}^{N}$-norm. It will always be clear from the context which norm is used. For $\zeta \in \mathbb{R}$, let $\zeta^{ \pm}=\max \{ \pm \zeta, 0\}$. Then for $u \in W_{0}^{1, p}(\Omega)$, we set $u^{ \pm}(\cdot)=u(\cdot)^{ \pm}$. We have $u^{ \pm} \in H_{0}^{1}(\Omega)$, $|u|=u^{+}+u^{-}$, and $u=u^{+}-u^{-}$. For a given measurable function $h: \Omega \times \mathbb{R} \rightarrow \mathbb{R}$ (e.g., a Carathéodory function), we set

$$
N_{h}(u)(\cdot)=h(\cdot, u(\cdot)) \quad \forall u \in W_{0}^{1, p}(\Omega)
$$

Finally, let $A \in \mathcal{L}\left(H_{0}^{1}(\Omega), H^{-1}(\Omega)\right)$ be the operator, defined by

$$
\langle A(u), y\rangle=\int_{\Omega}(\nabla u, \nabla y)_{\mathbb{R}^{N}} d z \quad \forall u, y \in H_{0}^{1}(\Omega)
$$

For the properties of the operator $A$ we refer to Gasiński and Papageorgiou [11, Proposition 3.1, page 852]. 


\section{Solutions of Constant Sign}

In this section, for $\lambda>0$ small, we generate four nontrivial smooth solutions of constant sign (two positive and two negative). To this end, we introduce the following modifications of the nonlinearities $g(z, \cdot)$ and $f(z, \cdot)$ :

$$
g_{ \pm}(z, \zeta)=g\left(z, \pm \zeta^{ \pm}\right), \quad \widehat{f}_{ \pm}(z, \zeta)=f\left(z, \pm \zeta^{ \pm}\right)+\widehat{c}_{1}\left( \pm \zeta^{ \pm}\right),
$$

with $\widehat{c}_{1}>0$ as in (2.14). These modifications are Carathéodory functions. We set

$$
G_{ \pm}(z, \zeta)=\int_{0}^{\zeta} g_{ \pm}(z, s) d s, \quad \widehat{F}_{ \pm}(z, \zeta)=\int_{0}^{\zeta} \widehat{f}_{ \pm}(z, s) d s
$$

and consider the $C^{1}$-functionals $\widehat{\varphi}_{\lambda}^{ \pm}: H_{0}^{1}(\Omega) \rightarrow \mathbb{R}$, defined by

$$
\widehat{\varphi}_{\lambda}^{ \pm}(u)=\frac{1}{2} \sigma(u)+\frac{\widehat{c}_{1}}{2}\|u\|_{2}^{2}-\lambda \int_{\Omega} G_{ \pm}(z, u(z))-\int_{\Omega} \widehat{F}_{ \pm}(z, u(z)) d z \quad \forall u \in H_{0}^{1}(\Omega) .
$$

Proposition 3.1. If hypotheses $H_{g}, H_{f}$, and $H_{0}$ hold and $\lambda>0$, then the functionals $\widehat{\varphi}_{\lambda}^{ \pm}$satisfy the Cerami condition.

Proof. We do the proof for $\widehat{\varphi}_{\lambda}^{+}$, the proof for $\widehat{\varphi}_{\lambda}^{-}$being similar.

So, let $\left\{u_{n}\right\}_{n \geqslant 1} \subseteq H_{0}^{1}(\Omega)$ be a sequence, such that

$$
\left|\widehat{\varphi}_{\lambda}^{+}\left(u_{n}\right)\right| \leqslant M_{1} \quad \forall n \geqslant 1,
$$

for some $M_{1}>0$ and

$$
\left(1+\left\|u_{n}\right\|\right)\left(\widehat{\varphi}_{\lambda}^{+}\right)^{\prime}\left(u_{n}\right) \longrightarrow 0 \quad \text { in } H^{-1}(\Omega) .
$$

From (3.5), we have

$$
\begin{aligned}
& \left|\left\langle A\left(u_{n}\right), h\right\rangle+\int_{\Omega}\left(\beta+\widehat{c}_{1}\right) u_{n} h d z-\lambda \int_{\Omega} g_{+}\left(z, u_{n}\right) h d z-\int_{\Omega} \widehat{f}_{+}\left(z, u_{n}\right) h d z\right| \\
& \quad \leqslant \frac{\varepsilon_{n}\|h\|}{1+\left\|u_{n}\right\|} \quad \forall h \in H_{0}^{1}(\Omega),
\end{aligned}
$$

with $\varepsilon_{n} \searrow 0$. In (3.6) we choose $h=-u_{n}^{-} \in H_{0}^{1}(\Omega)$. Then

$$
\left|\sigma\left(u_{n}^{-}\right)+\widehat{c}_{1}\left\|u_{n}^{-}\right\|_{2}^{2}\right| \leqslant \varepsilon_{n} \quad \forall n \geqslant 1,
$$

so

$$
\frac{1}{\widehat{c}_{0}}\left\|u_{n}^{-}\right\|^{2} \leqslant \varepsilon_{n} \quad \forall n \geqslant 1
$$


(see (2.14)), and hence

$$
u_{n}^{-} \longrightarrow 0 \text { in } H_{0}^{1}(\Omega)
$$

From (3.4) and (3.9), we have

$$
\sigma\left(u_{n}^{+}\right)-\lambda \int_{\Omega} 2 G_{+}\left(z, u_{n}^{+}\right) d z-\int_{\Omega} 2 F\left(z, u_{n}^{+}\right) d z \leqslant M_{2} \quad \forall n \geqslant 1,
$$

for some $M_{2}>0$. Also, if in (3.6) we choose $h=u_{n}^{+} \in H_{0}^{1}(\Omega)$, then

$$
-\sigma\left(u_{n}^{+}\right)+\lambda \int_{\Omega} g\left(z, u_{n}^{+}\right) u_{n}^{+} d z+\int_{\Omega} f\left(z, u_{n}^{+}\right) u_{n}^{+} d z \leqslant \varepsilon_{n} \quad \forall n \geqslant 1 .
$$

Adding (3.10) and (3.11), we obtain

$$
\int_{\Omega} \xi_{\lambda}\left(z, u_{n}^{+}\right) d z \leq M_{3} \quad \forall n \geqslant 1
$$

for some $M_{3}>0$ (see (2.29) for the definition of $\xi_{\curlywedge}$ ).

Claim 1. The sequence $\left\{u_{n}^{+}\right\}_{n \geqslant 1} \subseteq H_{0}^{1}(\Omega)$ is bounded.

Arguing by contradiction, suppose that the claim is not true. Then by passing to a subsequence if necessary, we may assume that

$$
\left\|u_{n}^{+}\right\| \longrightarrow+\infty
$$

Let

$$
y_{n}=\frac{u_{n}^{+}}{\left\|u_{n}^{+}\right\|} \quad \forall n \geqslant 1
$$

Then

$$
\left\|y_{n}\right\|=1 \quad \forall n \geqslant 1
$$

And so, passing to a subsequence if necessary, we may assume that

$$
\begin{gathered}
y_{n} \longrightarrow y \quad \text { weakly in } H_{0}^{1}(\Omega), \\
y_{n} \longrightarrow y \quad \text { in } L^{r}(\Omega) .
\end{gathered}
$$

If $y \neq 0$, then

$$
u_{n}^{+}(z) \longrightarrow+\infty \text { for almost all } z \in \Omega_{+}=\{y>0\}
$$


(recall that $y \geqslant 0$ ). So, by virtue of hypotheses $H_{g}$ (ii) and $H_{f}(\mathrm{ii})$, for almost all $z \in \Omega_{+}$, we have

$$
\begin{gathered}
\lim _{n \rightarrow+\infty} \frac{G\left(z, u_{n}^{+}(z)\right)}{\left\|u_{n}^{+}\right\|^{2}}=\lim _{n \rightarrow+\infty} \frac{G\left(z, u_{n}^{+}(z)\right)}{u_{n}^{+}(z)^{2}} y_{n}(z)^{2}=0, \\
\lim _{n \rightarrow+\infty} \frac{F\left(z, u_{n}^{+}(z)\right)}{\left\|u_{n}^{+}\right\|^{2}}=\lim _{n \rightarrow+\infty} \frac{F\left(z, u_{n}^{+}(z)\right)}{u_{n}^{+}(z)^{2}} y_{n}(z)^{2}=+\infty .
\end{gathered}
$$

Then Fatou's lemma implies that

$$
\lim _{n \rightarrow+\infty}\left(\lambda \int_{\Omega} \frac{G\left(z, u_{n}^{+}\right)}{\left\|u_{n}^{+}\right\|^{2}} d z+\int_{\Omega} \frac{F\left(z, u_{n}^{+}\right)}{\left\|u_{n}^{+}\right\|^{2}} d z\right)=+\infty
$$

But from (3.4) and (3.9), we have

$$
\lambda \int_{\Omega} G\left(z, u_{n}^{+}\right) d z+\int_{\Omega} F\left(z, u_{n}^{+}\right) d z \leqslant M_{4}+\left|\sigma\left(u_{n}^{+}\right)\right| \quad \forall n \geqslant 1
$$

for some $M_{4}>0$, so

$$
\lambda \int_{\Omega} \frac{G\left(z, u_{n}^{+}\right)}{\left\|u_{n}^{+}\right\|^{2}} d z+\int_{\Omega} \frac{F\left(z, u_{n}^{+}\right)}{\left\|u_{n}^{+}\right\|^{2}} d z \leqslant M_{5} \quad \forall n \geqslant 1
$$

for some $M_{5}>0$ (since the sequence $\left\{\sigma\left(y_{n}\right)\right\}_{n \geqslant 1}$ is bounded in $\mathbb{R}$ ). Comparing (3.20) and (3.22), we reach a contradiction.

So, we have $y=0$. We fix $\mu>0$ and set

$$
v_{n}=(2 \mu)^{1 / 2} y_{n} \quad \forall n \geqslant 1 .
$$

Evidently

$$
v_{n} \longrightarrow 0 \text { in } L^{r}(\Omega)
$$

(see (3.17)). Hence by Krasnoselskii's theorem (see Gasiński and Papageorgiou [12, Proposition 1.4.14, page 87] and hypotheses $H_{g}(i)$ and $\left.H_{f}(\mathrm{i})\right)$, we have

$$
\int_{\Omega} G\left(z, v_{n}(z)\right) d z \longrightarrow 0, \quad \int_{\Omega} F\left(z, v_{n}(z)\right) d z \longrightarrow 0 .
$$

Since $\left\|u_{n}^{+}\right\| \rightarrow+\infty$, we can find $n_{0} \geqslant 1$, such that

$$
0<(2 \mu)^{1 / 2} \frac{1}{\left\|u_{n}^{+}\right\|}<1 \quad \forall n \geqslant n_{0}
$$


Let $t_{n} \in[0,1]$ be such that

$$
\widehat{\varphi}_{\lambda}^{+}\left(t_{n} u_{n}^{+}\right)=\max _{0 \leqslant t \leqslant 1} \widehat{\varphi}_{\lambda}^{+}\left(t u_{n}^{+}\right)
$$

By virtue of (3.26), we have

$$
\begin{aligned}
\widehat{\varphi}_{\lambda}^{+}\left(t_{n} u_{n}^{+}\right) & \geqslant \widehat{\varphi}_{\lambda}^{+}\left(v_{n}\right) \\
& =2 \mu \sigma\left(y_{n}\right)-\lambda \int_{\Omega} G\left(z, v_{n}\right) d z-\int_{\Omega} F\left(z, v_{n}\right) d z \\
& =2 \mu+2 \mu \int_{\Omega} \beta y_{n}^{2} d z-\lambda \int_{\Omega} G\left(z, v_{n}\right) d z-\int_{\Omega} F\left(z, v_{n}\right) d z
\end{aligned}
$$

Note that

$$
\int_{\Omega} \beta y_{n}^{2} d z \rightarrow 0
$$

This fact together with (3.25) and (3.28) implies that

$$
\widehat{\varphi}_{\lambda}^{+}\left(t_{n} u_{n}^{+}\right) \geqslant \mu \quad \forall n \geqslant n_{1}
$$

for some $n_{1} \geqslant n_{0}$. Since $\mu>0$ is arbitrary, we infer that

$$
\widehat{\varphi}_{\lambda}^{+}\left(t_{n} u_{n}^{+}\right) \longrightarrow+\infty
$$

Note that

$$
\widehat{\varphi}_{\lambda}^{+}(0)=0, \quad \widehat{\varphi}_{\lambda}^{+}\left(u_{n}^{+}\right) \leqslant M_{6} \quad \forall n \geqslant 1,
$$

for some $M_{6}>0$ (see (3.4) and (3.9)).

Hence (3.31) implies that there exists $n_{2} \geqslant n_{1}$, such that

$$
t_{n} \in(0,1) \quad \forall n \geqslant n_{2} .
$$

And so from the choice of $t_{n}$, we have

$$
\left.\frac{d}{d t} \widehat{\varphi}_{\lambda}^{+}\left(t u_{n}^{+}\right)\right|_{t=t_{n}}=0 \quad \forall n \geqslant n_{2}
$$

so

$$
\left\langle\left(\widehat{\varphi}_{\lambda}^{+}\right)^{\prime}\left(t_{n} u_{n}\right), u_{n}\right\rangle=0 \quad \forall n \geqslant n_{2}
$$


thus

$$
\left\langle\left(\widehat{\varphi}_{\lambda}^{+}\right)^{\prime}\left(t_{n} u_{n}\right), t_{n} u_{n}\right\rangle=0 \quad \forall n \geqslant n_{2}
$$

and hence

$$
\sigma\left(t_{n} u_{n}^{+}\right)=\lambda \int_{\Omega} g\left(z, t_{n} u_{n}^{+}\right) t_{n} u_{n}^{+} d z+\int_{\Omega} f\left(z, t_{n} u_{n}^{+}\right) t_{n} u_{n}^{+} d z
$$

Hypothesis $H_{0}$ implies that

$$
\int_{\Omega} \xi_{\lambda}\left(z, t_{n} u_{n}^{+}\right) d z \leqslant \int_{\Omega} \xi_{\lambda}\left(z, u_{n}^{+}\right) d z+\left\|\vartheta_{\lambda}^{*}\right\|_{1} \quad \forall n \geqslant 1
$$

so

$$
2 \widehat{\varphi}_{\lambda}^{+}\left(t_{n} u_{n}^{+}\right) \leqslant \int_{\Omega} \xi_{\lambda}\left(z, u_{n}^{+}\right) d z+\left\|\vartheta_{\lambda}^{*}\right\|_{1} \quad \forall n \geqslant 1
$$

(see (3.37)), and thus

$$
2 \widehat{\varphi}_{\lambda}^{+}\left(t_{n} u_{n}^{+}\right) \leqslant M_{3}+\left\|\vartheta_{\lambda}^{*}\right\|_{1} \quad \forall n \geqslant 1
$$

(see (3.12)).

Comparing (3.31) and (3.40), we reach a contradiction. This proves the claim.

By virtue of the claim and (3.9), we have that the sequence $\left\{u_{n}\right\}_{n \geqslant 1} \subseteq H_{0}^{1}(\Omega)$ is bounded. So, we may assume that

$$
\begin{gathered}
u_{n} \longrightarrow u \quad \text { weakly in } H_{0}^{1}(\Omega), \\
u_{n} \longrightarrow u \text { in } L^{r}(\Omega) .
\end{gathered}
$$

In (3.6) we choose $h=u_{n}-u$, pass to the limit as $n \rightarrow+\infty$, and use (3.42). Then

$$
\lim _{n \rightarrow+\infty}\left\langle A\left(u_{n}\right), u_{n}-u\right\rangle=0,
$$

so

$$
\left\|u_{n}\right\|=\left\|\nabla u_{n}\right\|_{2} \longrightarrow\|\nabla u\|_{2}=\|u\|
$$

(see Gasiński and Papageorgiou [11, Proposition 3.1, page 852]), and thus

$$
u_{n} \longrightarrow u \quad \text { in } H_{0}^{1}(\Omega)
$$


(by the Kadec-Klee property of Hilbert spaces). This proves that $\widehat{\varphi}_{\lambda}^{+}$satisfies the Cerami condition.

Similarly we show that $\widehat{\varphi}_{\lambda}^{-}$also satisfies the Cerami condition.

Our aim is to apply Theorem 2.1 (the mountain pass theorem) to two functionals $\hat{\varphi}_{\lambda}^{+}$ and $\widehat{\varphi}_{\lambda}^{-}$. We have checked that both functionals satisfy the Cerami condition. So, it remains to show that they satisfy the mountain pass geometry as it is described in Theorem 2.1.

The next proposition is a crucial step in satisfying the mountain pass geometry for the two functionals $\widehat{\varphi}_{\lambda}^{+}$and $\widehat{\varphi}_{\lambda}^{-}$.

Proposition 3.2. If hypotheses $H_{g}$ and $H_{f}$ hold, then there exist $\lambda_{ \pm}^{*}>0$, such that for every $\lambda \in$ $\left(0, \lambda_{ \pm}^{*}\right)$, we can find $\rho_{\lambda}^{ \pm}>0$, such that

$$
\inf \left\{\widehat{\varphi}_{\lambda}^{ \pm}(u):\|u\|=\varphi_{\lambda}^{ \pm}\right\}=\widehat{\eta}_{\lambda}^{ \pm}>0 .
$$

Proof. Hypotheses $H_{g}$ (i) and (ii) imply that for a given $\varepsilon>0$, we can find $c_{4}=c_{4}(\varepsilon)>0$, such that

$$
g(z, \zeta) \leqslant \varepsilon \zeta+c_{4} \zeta^{q-1} \text { for almost all } z \in \Omega, \text { all } \zeta \geqslant 0
$$

so

$$
G(z, \zeta) \leqslant \frac{\varepsilon}{2} \zeta^{2}+\frac{c_{4}}{q} \zeta^{q} \text { for almost all } z \in \Omega, \text { all } \zeta \geqslant 0
$$

Similarly hypotheses $H_{f}(\mathrm{i})$ and (iii) imply that for a given $\varepsilon>0$, we can find $c_{5}=c_{5}(\varepsilon)>0$, such that

$$
f(z, \zeta) \leqslant\left(\eta_{0}(z)+\varepsilon\right) \zeta+c_{5} \zeta^{r-1} \text { for almost all } z \in \Omega, \text { all } \zeta \geqslant 0,
$$

so

$$
F(z, \zeta) \leqslant \frac{1}{2}\left(\eta_{0}(z)+\varepsilon\right) \zeta^{2}+\frac{c_{5}}{r} \zeta^{r} \text { for almost all } z \in \Omega, \text { all } \zeta \geqslant 0
$$

Then, for $u \in H_{0}^{1}(\Omega)$, we have

$$
\begin{aligned}
\widehat{\varphi}_{\lambda}^{+}(u)= & \frac{1}{2} \sigma(u)+\frac{\widehat{c}_{1}}{2}\|u\|_{2}^{2}-\lambda \int_{\Omega} G_{+}(z, u) d z-\int_{\Omega} \widehat{F}_{+}(z, u) d z \\
\geqslant & \frac{1}{2} \sigma\left(u^{+}\right)-\frac{1}{2} \int_{\Omega} \eta_{0}\left(u^{+}\right)^{2} d z-\frac{\varepsilon}{2}\left\|u^{+}\right\|_{2}^{2}-\frac{c_{5}}{r}\left\|u^{+}\right\|_{r}^{r} \\
& -\frac{\lambda \varepsilon}{2}\left\|u^{+}\right\|_{2}^{2}-\frac{\lambda c_{4}}{q}\left\|u^{+}\right\|_{q}^{q}+\frac{1}{2} \sigma\left(u^{-}\right)+\frac{\widehat{c}_{1}}{2}\left\|u^{-}\right\|_{2}^{2} \\
\geqslant & \frac{1}{2}\left(\widehat{c}-\frac{\varepsilon(\lambda+1)}{\widehat{\lambda}_{1}}\right)\left\|u^{+}\right\|^{2}+\frac{1}{2 \widehat{c}_{0}}\left\|u^{-}\right\|^{2}-c_{6}\left(\|u\|^{r}+\lambda\|u\|^{q}\right),
\end{aligned}
$$

for some $c_{6}>0$ (see (3.48), (3.50), (2.11), (2.14), and Lemma 2.2). 
Choosing $\varepsilon \in\left(0, \widehat{\lambda}_{1} \widehat{c} /(\lambda+1)\right)$, we have

$$
\begin{aligned}
\hat{\varphi}_{\lambda}^{+}(u) & \geqslant c_{7}\|u\|^{2}-c_{6}\left(\|u\|^{r}+\lambda\|u\|^{q}\right) \\
& =\left(c_{7}-c_{6}\left(\|u\|^{r-2}+\lambda\|u\|^{q-2}\right)\right)\|u\|^{2},
\end{aligned}
$$

for some $c_{7}>0$. Let

$$
\mu_{\lambda}(t)=t^{r-2}+\lambda t^{q-2} \quad \forall t>0 .
$$

Since $q<2<r$, we have

$$
\lim _{t \rightarrow 0^{+}} \mu_{\lambda}(t)=\lim _{r \rightarrow+\infty} \mu_{\lambda}(t)=+\infty
$$

Also $\mu_{\lambda}$ is continuous in $(0,+\infty)$. Therefore, we can find $t_{0} \in(0,+\infty)$, such that

$$
\mu_{\lambda}\left(t_{0}\right)=\inf _{t>0} \mu_{\lambda}(t)
$$

so

$$
\mu_{\lambda}^{\prime}\left(t_{0}\right)=0,
$$

and thus

$$
t_{0}=t_{0}(\lambda)=\left(\frac{\lambda(2-q)}{r-2}\right)^{1 /(r-q)}
$$

Evidently

$$
\mu_{\lambda}\left(t_{0}\right) \longrightarrow 0 \quad \text { as } \lambda \longrightarrow 0^{+} .
$$

Hence we can find $\lambda_{+}^{*}>0$, such that

$$
\mu_{\lambda}\left(t_{0}\right)<\frac{c_{7}}{c_{6}} \quad \forall \lambda \in\left(0, \lambda_{+}^{*}\right),
$$

so

$$
\inf \left\{\widehat{\varphi}_{\lambda}^{+}(u):\|u\|=\rho_{\lambda}^{+}=t_{0}(\lambda)\right\}=\widehat{\eta}_{\lambda}^{+}>0
$$

(see (3.52)). 

such that

Similarly, for $\widehat{\varphi}_{\lambda}^{-}$, we can find $\lambda_{-}^{*}>0$, such that for all $\lambda \in\left(0, \lambda_{-}^{*}\right)$ there exists $\varphi_{\lambda}^{-}>0$,

$$
\inf \left\{\widehat{\varphi}_{\lambda}^{-}(u):\|u\|=\rho_{\lambda}^{-}\right\}=\widehat{\eta}_{\lambda}^{-}>0
$$

With the next proposition we complete the mountain pass geometry for problem $\left((P)_{\lambda}\right)$.

Proposition 3.3. If hypotheses $H_{g}$ and $H_{f}$ hold, $\lambda>0$ and $\tilde{u} \in \operatorname{int} C_{+}$with $\|\tilde{u}\|_{2}=1$, then

$$
\widehat{\varphi}_{\lambda}^{ \pm}(t \tilde{u}) \longrightarrow-\infty \quad \text { as } t \longrightarrow \pm \infty
$$

Proof. By virtue of hypotheses $H_{g}(\mathrm{i})$ and (ii), for a given $\varepsilon>0$, we can find $c_{8}=c_{8}(\varepsilon)>0$, such that

$$
G(z, \zeta) \geqslant-\frac{\varepsilon}{2} \zeta^{2}-c_{8}, \quad \text { for almost all } z \in \Omega, \text { all } \zeta \in \mathbb{R}
$$

Similarly, hypotheses $H_{f}\left(\right.$ i) and (ii) imply that for any given $\xi>0$, we can find $c_{9}=c_{9}(\xi)>0$, such that

$$
F(z, \zeta) \geqslant \frac{\xi}{2} \zeta^{2}-c_{9}, \quad \text { for almost all } z \in \Omega, \text { all } \zeta \in \mathbb{R}
$$

Then, we have

$$
\begin{aligned}
\hat{\varphi}_{\lambda}^{+}(t \tilde{u}) & =\frac{t^{2}}{2} \sigma(\tilde{u})+\left(\frac{\lambda \varepsilon-\xi}{2}\right) t^{2}+c_{10} \\
& \leqslant \frac{t^{2}}{2}\left(\|\tilde{u}\|^{2}+\|\beta\|_{s}\|\tilde{u}\|_{2 s^{\prime}}+\lambda \varepsilon-\xi\right)+c_{10}
\end{aligned}
$$

for some $c_{10}>0$ (see (3.63), (3.64) and recall that $\tilde{u} \in \operatorname{int} C_{+},\|\tilde{u}\|_{2}=1$ and $\left.1 / s+1 / s^{\prime}=1\right)$.

Since $\xi>0$ is arbitrary, choosing

$$
\xi>\lambda \varepsilon+\|\tilde{u}\|^{2}+\|\beta\|_{s}\|\tilde{u}\|_{2 s^{\prime}}
$$

from (3.65), we infer that

$$
\widehat{\varphi}_{\lambda}^{+}(t \tilde{u}) \longrightarrow-\infty \quad \text { as } t \longrightarrow \pm \infty
$$

Now we are ready to produce the first two nontrivial smooth solutions of constant sign. In what follows, we set

$$
\lambda^{*}=\min \left\{\lambda_{-}^{*}, \lambda_{+}^{*}\right\}
$$


Proposition 3.4. If hypotheses $H_{g}, H_{f}$, and $H_{0}$ hold and $\lambda \in\left(0, \lambda^{*}\right]$, then problem $\left((P)_{\lambda}\right)$ has at least two nontrivial smooth solutions of constant sign:

$$
u_{0}, v_{0} \in C_{0}^{1}(\bar{\Omega}), \quad \text { with } v_{0}(z)<0<u_{0}(z) \quad \forall z \in \Omega \text {. }
$$

Proof. Propositions 3.1, 3.2, and 3.3 permit the application of the mountain pass theorem (see Theorem 2.1) for the functional $\widehat{\varphi}_{\lambda}^{+}$, and so we obtain $u_{0} \in H_{0}^{1}(\Omega)$, such that

$$
\begin{gathered}
\widehat{\varphi}_{\lambda}^{+}(0)=0<\widehat{\eta}_{\lambda}^{+} \leqslant \widehat{\varphi}_{\lambda}^{+}\left(u_{0}\right), \\
\left(\widehat{\varphi}_{\lambda}^{+}\right)^{\prime}\left(u_{0}\right)=0 .
\end{gathered}
$$

From (3.70), we see that $u_{0} \neq 0$. From (3.71), we have

$$
A\left(u_{0}\right)+\beta u_{0}+\widehat{c}_{1} u_{0}=\lambda N_{g_{+}}\left(u_{0}\right)+N_{\widehat{f}_{+}}\left(u_{0}\right) .
$$

On (3.72) we act with $-u_{0}^{-} \in H_{0}^{1}(\Omega)$ and obtain

$$
\sigma\left(u_{0}^{-}\right)+\widehat{c}_{1}\left\|u_{0}^{-}\right\|_{2}^{2}=0,
$$

so

$$
\frac{1}{\widehat{c}_{0}}\left\|u_{0}^{-}\right\|^{2} \leqslant 0
$$

(see (2.14)); hence $u_{0} \geqslant 0, u_{0} \neq 0$.

Therefore (3.72) becomes

$$
A\left(u_{0}\right)+\beta u_{0}=\lambda N_{g}\left(u_{0}\right)+N_{f}\left(u_{0}\right)
$$

so

$$
\begin{gathered}
-\Delta u_{0}(z)+\beta(z) u_{0}(z)=\lambda g\left(z, u_{0}(z)\right)+f\left(z, u_{0}(z)\right) \text { in } \Omega, \\
\left.u_{0}\right|_{\partial \Omega}=0 .
\end{gathered}
$$

From the regularity theory for Dirichlet problems (see Struwe [13, pp. 217-219]), we have that $u_{0} \in C_{0}^{1}(\bar{\Omega})$. Moreover, invoking the weak Harnack inequality of Pucci and Serrin [14, page 154], we have that $u_{0}(z)>0$ for all $z \in \Omega$.

Similarly working with $\widehat{\varphi}_{\lambda}^{-}$, this time we obtain a nontrivial smooth negative solution $v_{0} \in C_{0}^{1}(\bar{\Omega})$ with $v_{0}(z)<0$ for all $z \in \Omega$.

We can improve the conclusion of this proposition by strengthening the condition on the potential $\beta$ :

$$
\underline{H_{\beta}}: \beta \in L^{s}(\Omega) \text { with } s>N / 2 \text { and } \beta^{+} \in L^{\infty}(\Omega)_{+} .
$$


Remark 3.5. So, the potential function is bounded from above but in general can be unbounded from below.

Proposition 3.6. If hypotheses $H_{g}, H_{f}, H_{0}$, and $H_{\beta}$ hold and $\lambda \in\left(0, \lambda^{*}\right)$, then problem $\left((P)_{\lambda}\right)$ has at least two nontrivial smooth solutions of constant sign:

$$
u_{0} \in \operatorname{int} C_{+}, \quad v_{0} \in-\operatorname{int} C_{+} .
$$

Proof. From Proposition 3.4, we already have two solutions:

$$
u_{0}, v_{0} \in C_{0}^{1}(\bar{\Omega}), \quad \text { with } v_{0}(z)<0<u_{0}(z) \quad \forall z \in \Omega
$$

We have

$$
\begin{aligned}
-\Delta u_{0}(z)+\beta(z) u_{0}(z) & =\lambda g\left(z, u_{0}(z)\right)+f\left(z, u_{0}(z)\right) \\
& \geqslant f\left(z, u_{0}(z)\right) \text { for almost all } z \in \Omega
\end{aligned}
$$

(see hypothesis $H_{g}\left(\right.$ iii)). Let $\varrho=\left\|u_{0}\right\|_{\infty}$ and let $\widehat{\gamma}_{\rho}>0$ be as postulated by hypothesis $H_{f}(\mathrm{iv})$. Then from (3.79), we have

$$
-\Delta u_{0}(z)+\left(\beta(z)+\widehat{\gamma}_{\rho}\right) u_{0}(z) \geqslant f\left(z, u_{0}(z)\right)+\widehat{\gamma}_{\rho} u_{0}(z), \quad \text { for almost all } z \in \Omega,
$$

so

$$
\Delta u_{0}(z) \leqslant\left(\left\|\beta^{+}\right\|_{\infty}+\widehat{\gamma}_{\rho}\right) u_{0}(z) \text { for almost all } z \in \Omega
$$

and thus $u_{0} \in$ int $C_{+}$(see Vázquez [15] and Pucci and Serrin [14, page 120]).

Similarly for the negative solution $v_{0}$.

To continue and produce additional nontrivial smooth solutions of constant sign, we need to keep hypotheses $H_{\beta}$.

Proposition 3.7. If hypotheses $H_{g}, H_{f}, H_{0}$ and $H_{\beta}$ hold and $\lambda \in\left(0, \lambda^{*}\right)$, then problem $\left((P)_{\lambda}\right)$ has at least four nontrivial smooth solutions of constant sign:

$$
\begin{array}{cc}
u_{0}, \widehat{u} \in \operatorname{int} C_{+}, & \widehat{u}-u_{0} \in \operatorname{int} C_{+} \\
v_{0}, \widehat{v} \in-\operatorname{int} C_{+}, & v_{0}-\widehat{v} \in \operatorname{int} C_{+} .
\end{array}
$$

Proof. From Proposition 3.6, we already have two solutions:

$$
u_{0} \in \operatorname{int} C_{+}, \quad v_{0} \in-\operatorname{int} C_{+} .
$$


We introduce the following truncation perturbation of the reaction of the problem $\left((P)_{\mathcal{\Lambda}}\right)$ :

$$
h_{\lambda}^{+}(z, \zeta)= \begin{cases}\lg \left(z, u_{0}(z)\right)+f\left(z, u_{0}(z)\right)+\widehat{c}_{1} u_{0}(z) & \text { if } \zeta \leqslant u_{0}(z) \\ \lg (z, \zeta)+f(z, \zeta)+\widehat{c}_{1} \zeta & \text { if } u_{0}(z)<\zeta\end{cases}
$$

This is a Carathéodory function. We set

$$
H_{\lambda}^{+}(z, \zeta)=\int_{0}^{\zeta} h_{\lambda}^{+}(z, s) d s
$$

and consider the $C^{1}$-functional $\psi_{\lambda}^{+}: H_{0}^{1}(\Omega) \rightarrow \mathbb{R}$, defined by

$$
\psi_{\lambda}^{+}(u)=\frac{1}{2} \sigma(u)+\frac{\widehat{c}_{1}}{2}\|u\|_{2}^{2}-\int_{\Omega} H_{\lambda}^{+}(z, u(z)) d z \quad \forall u \in H_{0}^{1}(\Omega)
$$

Claim 2. We have $K_{\psi_{\perp}^{+}} \subseteq\left[u_{0}\right)$, where

$$
\left[u_{0}\right)=\left\{u \in H_{0}^{1}(\Omega): u_{0}(z) \leqslant u(z) \text { for almost all } z \in \Omega\right\}
$$

Let $\tilde{u} \in K_{\psi_{\lambda}^{+}}$. Then

$$
A(\tilde{u})+\left(\beta+\widehat{c}_{1}\right) \tilde{u}=N_{h_{\lambda}^{+}}(\tilde{u}) .
$$

On (3.88) we act with $\left(u_{0}-\tilde{u}\right)^{+} \in H_{0}^{1}(\Omega)$. Then

$$
\begin{aligned}
\langle A(\tilde{u}), & \left.\left(u_{0}-\tilde{u}\right)^{+}\right\rangle+\int_{\Omega}\left(\beta+\widehat{c}_{1}\right) \tilde{u}\left(u_{0}-\tilde{u}\right)^{+} d z \\
= & \int_{\Omega} h_{\lambda}^{+}(z, \tilde{u})\left(u_{0}-\tilde{u}\right)^{+} d z \\
= & \int_{\Omega}\left(\lambda g\left(z, u_{0}\right)+f\left(z, u_{0}\right)+\widehat{c}_{1} u_{0}\right)\left(u_{0}-\tilde{u}\right)^{+} d z \\
= & \left\langle A\left(u_{0}\right),\left(u_{0}-\widehat{u}\right)^{+}\right\rangle+\int_{\Omega}\left(\beta+\widehat{c}_{1}\right) u_{0}\left(u_{0}-\tilde{u}\right)^{+} d z
\end{aligned}
$$

(see (3.84)), so

$$
\left\langle A\left(u_{0}-\tilde{u}\right),\left(u_{0}-\tilde{u}\right)^{+}\right\rangle+\int_{\Omega} \beta\left(u_{0}-\tilde{u}\right)\left(u_{0}-\tilde{u}\right)^{+} d z+\widehat{\mathrm{c}}_{1}\left\|\left(u_{0}-\tilde{u}\right)^{+}\right\|_{2}^{2}=0,
$$

thus

$$
\sigma\left(\left(u_{0}-\tilde{u}\right)^{+}\right)+\widehat{c}_{1}\left\|\left(u_{0}-\tilde{u}\right)^{+}\right\|_{2}^{2}=0
$$


hence

$$
\frac{1}{\widehat{c}_{0}}\left\|\left(u_{0}-\tilde{u}\right)^{+}\right\|^{2} \leqslant 0
$$

(see $(2.14)$ ), and so finally $u_{0} \leqslant \tilde{u}$. This proves Claim 2 .

Claim 3. We may assume that $u_{0}$ is a local minimizer of $\psi_{\lambda}^{+}$.

Let $\mu \in\left(\lambda, \lambda^{*}\right)$, and consider problem $(P)_{\mu}$. As we did in the proof of Proposition 3.4, via the mountain pass theorem, we obtain a nontrivial smooth positive solution $u_{\mu}$, and by virtue of the strong maximum principle, we have $u_{\mu} \in \operatorname{int} C_{+}$(see the proof of Proposition 3.7). Then

$$
\begin{array}{r}
-\Delta u_{\mu}(z)+\beta(z) u_{\mu}(z)=\mu g\left(z, u_{\mu}(z)\right)+f\left(z, u_{\mu}(z)\right) \\
\geqslant \lambda g\left(z, u_{\mu}(z)\right)+f\left(z, u_{\mu}(z)\right) \quad \text { for almost all } z \in \Omega
\end{array}
$$

(see $H_{g}$ (iii) and recall that $\lambda<\mu$ ).

We consider the following truncation perturbation of the reaction of problem $\left((P)_{\lambda}\right)$ :

$$
\gamma_{\lambda}^{+}(z, \zeta)= \begin{cases}0 & \text { if } \zeta<0, \\ \lg (z, \zeta)+f(z, \zeta)+\widehat{c}_{1} \zeta & \text { if } 0 \leqslant \zeta \leqslant u_{\mu}(z) \\ \lg \left(z, u_{\mu}(z)\right)+f\left(z, u_{\mu}(z)\right)+\widehat{c}_{1} u_{\mu}(z) & \text { if } u_{\mu}(z)<\zeta .\end{cases}
$$

This is a Carathéodory function. We set

$$
\Gamma_{\lambda}^{+}(z, \zeta)=\int_{0}^{\zeta} r_{\lambda}^{+}(z, s) d s
$$

and consider the $C^{1}$-functional $\widehat{\psi}_{\lambda}^{+}: H_{0}^{1}(\Omega) \rightarrow \mathbb{R}$, defined by

$$
\widehat{\psi}_{\lambda}^{+}(u)=\frac{1}{2} \sigma(u)+\frac{\widehat{c}_{1}}{2}\|u\|_{2}^{2}-\int_{\Omega} \Gamma_{\lambda}^{+}(z, u(z)) d z \quad \forall u \in H_{0}^{1}(\Omega) .
$$

From (3.94), it is clear that $\widehat{\psi}_{\lambda}^{+}$is coercive. Also, using the Sobolev embedding theorem, we check that $\widehat{\psi}_{\lambda}^{+}$is sequentially weakly lower semicontinuous. So, by the Weierstrass theorem, we can find $u_{\curlywedge} \in H_{0}^{1}(\Omega)$, such that

$$
\widehat{\psi}_{\lambda}^{+}\left(u_{\curlywedge}\right)=\inf _{u \in H_{0}^{1}(\Omega)} \widehat{\psi}_{\lambda}^{+}(u)
$$

By virtue of hypothesis $H_{f}(\mathrm{iii})$, we can find $c_{11}>0$ and $\widehat{\delta}_{0}>0$, such that

$$
F(z, \zeta) \geqslant-\frac{c_{11}}{2} \zeta^{2} \quad \text { for almost all } z \in \Omega, \text { all }|\zeta| \leqslant \widehat{\delta}_{0}
$$


Let $\tilde{u} \in$ int $C_{+}$and let $t \in(0,1)$ be small, such that

$$
t \tilde{u} \leqslant u_{\mu}, \quad t \tilde{u}(z) \leqslant \widehat{\delta}_{0} \quad \forall z \in \bar{\Omega}
$$

(recall that $u_{\mu} \in \operatorname{int} C_{+}$). Then, we have

$$
\begin{aligned}
\widehat{\psi}_{\lambda}^{+}(t \tilde{u}) & =\frac{t^{2}}{2} \sigma(\tilde{u})+\frac{t^{2} \widehat{c}_{1}}{2}\left\|\tilde{u}^{-}\right\|_{2}^{2}-\lambda \int_{\Omega} G(z, t \tilde{u}) d z-\int_{\Omega} F(z, t \tilde{u}) d z \\
& \leqslant \frac{t^{2}}{2}\left(\sigma(\tilde{u})+\left(\widehat{c}_{1}+c_{11}\right)\|\tilde{u}\|_{2}^{2}\right)-\frac{\lambda t^{q} c_{1}}{q}\|\tilde{u}\|_{q}^{q}
\end{aligned}
$$

(see (3.94), hypothesis $H_{g}$ (iii), and (3.98)). Since $q<2$, by choosing $t \in(0,1)$ even smaller if necessary, we have

$$
\widehat{\psi}_{\lambda}^{+}(t \tilde{u})<0
$$

so

$$
\widehat{\psi}_{\lambda}^{+}\left(u_{\curlywedge}\right)<0=\widehat{\psi}_{\lambda}^{+}(0)
$$

(see (3.97)); hence $u_{\curlywedge} \neq 0$.

From (3.97), we have

$$
\left(\widehat{\varphi}_{\lambda}^{+}\right)^{\prime}\left(u_{\curlywedge}\right)=0
$$

so

$$
A\left(u_{\curlywedge}\right)+\left(\beta+\widehat{c}_{1}\right) u_{\lambda}=N_{\gamma_{\lambda}^{+}}\left(u_{\curlywedge}\right)
$$

Acting on (3.104) with $-u_{\lambda}^{-} \in H_{0}^{1}(\Omega)$, we obtain that $u_{\lambda} \geqslant 0, u_{\lambda} \neq 0$. Also, acting on (3.104) with $\left(u_{\lambda}-u_{\mu}\right)^{+} \in H_{0}^{1}(\Omega)$, we have

$$
\begin{aligned}
& \left\langle A\left(u_{\curlywedge}\right),\left(u_{\lambda}-u_{\mu}\right)^{+}\right\rangle+\int_{\Omega}\left(\beta+\widehat{c}_{1}\right) u_{\curlywedge}\left(u_{\curlywedge}-u_{\mu}\right)^{+} d z \\
& \quad=\int_{\Omega} r_{\lambda}^{+}\left(z, u_{\curlywedge}\right)\left(u_{\curlywedge}-u_{\mu}\right)^{+} d z \\
& \quad=\int_{\Omega}\left(\lambda g\left(z, u_{\mu}\right)+f\left(z, u_{\mu}\right)+\widehat{c}_{1} u_{\mu}\right)\left(u_{\curlywedge}-u_{\mu}\right)^{+} d z \\
& \quad \leqslant\left\langle A\left(u_{\mu}\right),\left(u_{\curlywedge}-u_{\mu}\right)^{+}\right\rangle+\int_{\Omega}\left(\beta+\widehat{c}_{1}\right) u_{\mu}\left(u_{\lambda}-u_{\mu}\right)^{+} d z
\end{aligned}
$$

(see (3.94) and (3.93)), so

$$
\left\langle A\left(u_{\curlywedge}-u_{\mu}\right),\left(u_{\curlywedge}-u_{\mu}\right)^{+}\right\rangle+\int_{\Omega}\left(\beta+\widehat{c}_{1}\right)\left(u_{\lambda}-u_{\mu}\right)\left(u_{\curlywedge}-u_{\mu}\right)^{+} d z \leqslant 0,
$$


thus

$$
\sigma\left(\left(u_{\curlywedge}-u_{\mu}\right)^{+}\right)+\widehat{c}_{1}\left\|\left(u_{\curlywedge}-u_{\mu}\right)^{+}\right\|_{2}^{2} \leqslant 0,
$$

hence

$$
\frac{1}{\widehat{c}_{0}}\left\|\left(u_{\curlywedge}-u_{\mu}\right)^{+}\right\|^{2} \leqslant 0
$$

(see (2.14)) and so finally

$$
u_{\lambda} \leqslant u_{\mu}
$$

So, we have proved that

$$
u_{\lambda} \in\left[0, u_{\mu}\right]=\left\{u \in H_{0}^{1}(\Omega): 0 \leqslant u(z) \leqslant u_{\mu}(z) \text { for almost all } z \in \Omega\right\} .
$$

Hence (3.104) becomes

$$
A\left(u_{\curlywedge}\right)+\beta u_{\curlywedge}=\lambda N_{g}\left(u_{\curlywedge}\right)+N_{f}\left(u_{\curlywedge}\right)
$$

(see (3.94)), so

$$
\begin{aligned}
-\Delta u_{\lambda}(z)+\beta(z) u_{\lambda}(z)= & \lambda g\left(z, u_{\lambda}(z)\right)+f\left(z, u_{\lambda}(z)\right) \text { in } \Omega, \\
& \left.u_{\lambda}\right|_{\partial \Omega}=0,
\end{aligned}
$$

thus $u_{\lambda} \in \operatorname{int} C_{+}$(as in the proof of Proposition 3.6) and it is a solution of $\left((P)_{\lambda}\right)$.

If $u_{\lambda} \neq u_{0}$, then this is the desired second nontrivial positive smooth solution of $\left((P)_{\lambda}\right)$.

So, we may assume that $u_{\lambda}=u_{0}$ and that there is no other solution of $\left((P)_{\lambda}\right)$ in the order interval

$$
\left[u_{0}, u_{\curlywedge}\right]=\left\{u \in H_{0}^{1}(\Omega): u_{0}(z) \leqslant u(z) \leqslant u_{\mu}(z) \text { for almost all } z \in \Omega\right\} .
$$

We introduce the following truncation of $\gamma_{\lambda}^{+}(z, \cdot)$ :

$$
\tilde{r}_{\lambda}^{+}(z, \zeta)= \begin{cases}r_{\lambda}^{+}\left(z, u_{0}(z)\right) & \text { if } \zeta<u_{0}(z) \\ \gamma_{\lambda}^{+}(z, \zeta) & \text { if } u_{0}(z) \leqslant \zeta\end{cases}
$$

This is a Carathéodory function. We set

$$
\widetilde{\Gamma}_{\lambda}^{+}(z, \zeta)=\int_{0}^{\zeta} \tilde{\gamma}_{\lambda}^{+}(z, s) d s
$$


and consider the $C^{1}$-functional $\tilde{\varphi}_{\lambda}^{+}: H_{0}^{1}(\Omega) \rightarrow \mathbb{R}$, defined by

$$
\tilde{\psi}_{\lambda}^{+}(u)=\frac{1}{2} \sigma(u)+\frac{\widehat{c}_{1}}{2}\|u\|_{2}^{2}-\int_{\Omega} \widetilde{\Gamma}_{\lambda}^{+}(z, u(z)) d z \quad \forall u \in H_{0}^{1}(\Omega) .
$$

From (3.94) and (3.114), it follows that $\tilde{\psi}_{\lambda}^{+}$is coercive. Also, it is sequentially weakly lower semicontinuous. Hence, we can find $\tilde{u}_{\curlywedge} \in H_{0}^{1}(\Omega)$, such that

$$
\tilde{\psi}_{\lambda}^{+}\left(\tilde{u}_{\curlywedge}\right)=\inf _{u \in H_{0}^{1}(\Omega)} \tilde{\psi}_{\curlywedge}^{+}(u)
$$

so

$$
\left(\tilde{\psi}_{\lambda}^{+}\right)^{\prime}\left(\tilde{u}_{\lambda}\right)=0
$$

and thus

$$
A\left(\tilde{u}_{\lambda}\right)+\left(\beta+\widehat{c}_{1}\right) \tilde{u}_{\lambda}=N_{\tilde{r}_{\lambda}^{+}}\left(\tilde{u}_{\lambda}\right) .
$$

As before, acting on (3.119) with $\left(u_{0}-\widetilde{u}_{\lambda}\right)^{+} \in H_{0}^{1}(\Omega)$ and with $\left(\tilde{u}_{\lambda}-u_{\mu}\right)^{+} \in H_{0}^{1}(\Omega)$, we show that $\tilde{u}_{\lambda} \in\left[u_{0}, u_{\mu}\right]$. Then, from (3.94) and (3.114), it follows that

$$
A\left(\tilde{u}_{\curlywedge}\right)+\beta \tilde{u}_{\curlywedge}=\lambda N_{g}\left(\tilde{u}_{\lambda}\right)+N_{f}\left(\tilde{u}_{\lambda}\right)
$$

so $\tilde{u}_{\lambda} \in \operatorname{int} C_{+}$is a solution of $\left((P)_{\lambda}\right)$ in $\left[u_{0}, u_{\mu}\right]$, hence $\tilde{u}_{\curlywedge}=u_{0}$.

Let $\rho=\left\|u_{0}\right\|_{\infty}$ and let $\gamma_{\rho}>0$ and $\hat{\gamma}_{\rho}>0$ be as postulated by hypotheses $H_{g}($ iv $)$ and $H_{f}(\mathrm{iv})$, respectively. We have

$$
\begin{aligned}
-\Delta & u_{0}(z)+\left(\beta(z)+\widehat{\gamma}_{\rho}\right) u_{0}(z)+\lambda \gamma_{\rho} u_{0}(z)^{q-1} \\
& =\lambda g\left(z, u_{0}(z)\right)+\lambda \gamma_{\rho} u_{0}(z)^{q-1}+f\left(z, u_{0}(z)\right)+\widehat{\gamma}_{\rho} u_{0}(z) \\
& \leqslant \lambda g\left(z, u_{\mu}(z)\right)+\lambda \gamma_{\rho} u_{\mu}(z)^{q-1}+f\left(z, u_{\mu}(z)\right)+\widehat{\gamma}_{\rho} u_{\mu}(z) \\
& \leqslant \mu g\left(z, u_{\mu}(z)\right)+\mu \gamma_{\rho} u_{\mu}(z)^{q-1}+f\left(z, u_{\mu}(z)\right)+\widehat{\gamma}_{\rho} u_{\mu}(z) \\
& =-\Delta u_{\mu}(z)+\left(\beta(z)+\widehat{\gamma}_{\rho}\right) u_{\mu}(z)+\mu \gamma_{\rho} u_{\mu}(z)^{q-1} \text { for almost all } z \in \Omega
\end{aligned}
$$

(see $H_{g}$ (iv) and (iii), $H_{f}$ (iv) and recall that $u_{0} \leqslant u_{\mu}$ and $\lambda<\mu$ ), so there exists $c_{12}>0$, such that

$$
\Delta\left(u_{\mu}-u_{0}\right)(z) \leqslant\left(\left\|\beta^{+}\right\|_{\infty}+\widehat{\gamma}_{\rho}\right)\left(u_{\mu}-u_{0}\right)(z)+\mu \gamma_{\rho} c_{12}\left(u_{\mu}-u_{0}\right)(z)
$$

for almost all $z \in \Omega$ (recall that the function $\zeta \mapsto \zeta^{q-1}$ is locally Lipschitz); thus

$$
u_{\mu}-u_{0} \in \text { int } C_{+}
$$


(see Struwe [13] and Pucci and Serrin [14, page 120]). So, we have that

$$
u_{\lambda}=u_{0} \in \operatorname{int}_{C_{0}^{1}(\bar{\Omega})}\left[0, u_{\mu}\right]
$$

Note that

$$
\left.\tilde{\psi}_{\lambda}^{+}\right|_{\left[0, u_{\mu}\right]}=\left.\psi_{\lambda}^{+}\right|_{\left[0, u_{\mu}\right]}
$$

(see (3.94) and (3.114)), so

$$
u_{0} \text { is alocal } C_{0}^{1}(\bar{\Omega}) \text {-minimizer of } \psi_{\lambda}^{+}
$$

(see (3.124)), and thus

$$
u_{0} \text { is alocal } H_{0}^{1}(\Omega) \text {-minimizer of } \psi_{\lambda}^{+}
$$

(Brézis and Nirenberg [16]). This proves Claim 3.

By virtue of Claim 3, as in Gasiński and Papageorgiou [17, proof of Theorem 3.4], we can find $\rho_{\curlywedge} \in(0,1)$ small, such that

$$
\psi_{\lambda}^{+}\left(u_{0}\right)<\inf \left\{\psi_{\lambda}^{+}(u):\left\|u-u_{0}\right\|=\rho_{\lambda}\right\}=\eta_{\lambda}^{+} .
$$

As in Proposition 3.4, for $\tilde{u} \in$ int $C_{+}$with $\|\tilde{u}\|_{2}=1$, we have

$$
\psi_{\lambda}^{+}(t \tilde{u}) \longrightarrow-\infty \text { as } t \longrightarrow+\infty \text {. }
$$

Note that $\widehat{\varphi}_{\lambda}^{+}=\psi_{\lambda}^{+}-\xi_{\lambda}^{+}$with $\xi_{\lambda}^{+} \in \mathbb{R}$. Hence by virtue of Proposition 3.1, $\psi_{\lambda}^{+}$satisfies the Cerami condition. This fact together with (3.128) and (3.129) permits the use of the mountain pass theorem (see Theorem 2.1). So, we can find $\widehat{u} \in H_{0}^{1}(\Omega)$, such that

$$
\begin{gathered}
\psi_{\lambda}^{+}\left(u_{0}\right)<\eta_{\lambda}^{+} \leqslant \psi_{\lambda}^{+}(\widehat{u}), \\
\left(\psi_{\lambda}^{+}\right)^{\prime}(\widehat{u})=0 .
\end{gathered}
$$

From (3.130) we have $\widehat{u} \neq u_{0}$. From (3.131) and Claim 2, we have that

$$
u_{0} \leqslant \widehat{u}
$$

Hence

$$
\mathrm{A}(\widehat{u})+\beta \widehat{u}=\lambda N_{g}(\widehat{u})+N_{f}(\widehat{u})
$$

(see (3.84)), and so $\widehat{u} \in \operatorname{int} C_{+}$solves problem $\left((P)_{\curlywedge}\right)$. 
Moreover, as before, using the strong maximum principle (see Vázquez [15] and Pucci and Serrin [14, page 120]), we have

$$
\widehat{u}-u_{0} \in \operatorname{int} C_{+} .
$$

In a similar way, using $v_{0} \in-$ int $C_{+}$, we define

$$
h_{\lambda}^{-}(z, \zeta)= \begin{cases}\lambda g(z, \zeta)+f(z, \zeta)+\widehat{c}_{1} \zeta & \text { if } \zeta<v_{0}(z) \\ \lambda g\left(z, v_{0}(z)\right)+f\left(z, v_{0}(z)\right)+\widehat{c}_{1} v_{0}(z) & \text { if } v_{0}(z) \leqslant \zeta\end{cases}
$$

This is a Carathéodory function. We set

$$
H_{\lambda}^{-}(z, \zeta)=\int_{0}^{\zeta} h_{\lambda}^{-}(z, s) d s
$$

and consider the $C^{1}$-functional $\psi_{\lambda}^{-}: H_{0}^{1}(\Omega) \rightarrow \mathbb{R}$, defined by

$$
\psi_{\lambda}^{-}(u)=\frac{1}{2} \sigma(u)+\frac{\widehat{c}_{1}}{2}\|u\|_{2}^{2}-\int_{\Omega} H_{\lambda}^{-}(z, u(z)) d z \quad \forall u \in H_{0}^{1}(\Omega)
$$

Reasoning as above, using this time $\psi_{\lambda}^{-}$, we obtain a second negative smooth solution $\widehat{v} \in$ -int $C_{+}$of problem $\left((P)_{\lambda}\right)$, such that

$$
v_{0}-\widehat{v} \in \operatorname{int} C_{+} \text {. }
$$

\section{Five Solutions}

In this section, we prove two multiplicity theorems, establishing five nontrivial smooth solutions when $\lambda \in\left(0, \lambda^{*}\right)$. In the second multiplicity theorem, we provide sign information for all the solutions (i.e., we show that the fifth solution is actually nodal).

Theorem 4.1. If hypotheses $H_{g}, H_{f}, H_{0}$, and $H_{\beta}$ hold and $\lambda \in\left(0, \lambda^{*}\right)$, then problem $\left((P)_{\lambda}\right)$ has at least five nontrivial smooth solutions:

$$
\begin{gathered}
u_{0}, \widehat{u} \in \operatorname{int} C_{+}, \quad \widehat{u}-u_{0} \in \operatorname{int} C_{+}, \quad v_{0}, \widehat{v} \in-\operatorname{int} C_{+}, \quad v_{0}-\widehat{v} \in \operatorname{int} C_{+} \\
y_{0} \in C_{0}^{1}(\bar{\Omega}), \quad u_{0}-y_{0} \in \operatorname{int} C_{+}, \quad y_{0}-v_{0} \in \operatorname{int} C_{+} .
\end{gathered}
$$

Proof. From Proposition 3.7, we already have four nontrivial smooth solutions of constant sign:

$$
u_{0}, \widehat{u} \in \operatorname{int} C_{+}, \quad \widehat{u}-u_{0} \in \operatorname{int} C_{+}, v_{0}, \quad \widehat{v} \in-\operatorname{int} C_{+}, \quad v_{0}-\widehat{v} \in \operatorname{int} C_{+} .
$$


We consider the following truncation perturbation of the reaction of problem $\left((P)_{\curlywedge}\right)$ :

$$
h_{\lambda}^{*}(z, \zeta)= \begin{cases}\operatorname{\lambda g}\left(z, v_{0}(z)\right)+f\left(z, v_{0}(z)\right)+\widehat{c}_{1} v_{0}(z) & \text { if } \zeta<v_{0}(z), \\ \lg (z, \zeta)+f(z, \zeta) & \text { if } v_{0}(z) \leqslant \zeta \leqslant u_{0}(z) \\ \lg \left(z, u_{0}(z)\right)+f\left(z, u_{0}(z)\right)+\widehat{c}_{1} u_{0}(z) & \text { if } u_{0}(z)<\zeta .\end{cases}
$$

This is a Carathéodory function. We set

$$
H_{\curlywedge}^{*}(z, \zeta)=\int_{0}^{\zeta} h_{\lambda}^{*}(z, s) d s
$$

and consider the $C^{1}$-functional $\psi_{\lambda}^{*}: H_{0}^{1}(\Omega) \rightarrow \mathbb{R}$, defined by

$$
\psi_{\lambda}^{*}(u)=\frac{1}{2} \sigma(u)+\frac{\widehat{c}_{1}}{2}\|u\|_{2}^{2}-\int_{\Omega} H_{\lambda}^{*}(z, u(z)) d z \quad \forall u \in H_{0}^{1}(\Omega)
$$

From (4.3), it follows that $\psi_{\lambda}^{*}$ is coercive. Also, it is sequentially weakly lower semicontinuous. So, we can find $y_{0} \in H_{0}^{1}(\Omega)$, such that

$$
\psi_{\lambda}^{*}\left(y_{0}\right)=\inf _{u \in H_{0}^{1}(\Omega)} \psi_{\lambda}^{*}(u)
$$

As in the proof of Proposition 3.7, using (3.98), we show that

$$
\psi_{\lambda}^{*}\left(y_{0}\right)<0=\psi_{\lambda}^{*}(0)
$$

hence $y_{0} \neq 0$. From (4.6), we have

$$
\left(\psi_{\lambda}^{*}\right)^{\prime}\left(y_{0}\right)=0
$$

so

$$
A\left(y_{0}\right)+\left(\beta+\widehat{c}_{1}\right) y_{0}=N_{h_{\lambda}^{*}}\left(y_{0}\right)
$$

On (4.9) we act with $\left(y_{0}-u_{0}\right)^{+} \in H_{0}^{1}(\Omega)$. Then

$$
\begin{aligned}
& \left\langle A\left(y_{0}\right),\left(y_{0}-u_{0}\right)^{+}\right\rangle+\int_{\Omega}\left(\beta+\widehat{c}_{1}\right) y_{0}\left(y_{0}-u_{0}\right)^{+} d z \\
& \quad=\int_{\Omega}\left(\lambda g\left(z, u_{0}\right)+f\left(z, u_{0}\right)+\widehat{c}_{1} u_{0}\right)\left(y_{0}-u_{0}\right)^{+} d z \\
& =\left\langle A\left(u_{0}\right),\left(y_{0}-u_{0}\right)^{+}\right\rangle+\int_{\Omega}(\beta+\widehat{c}) u_{0}\left(y_{0}-u_{0}\right)^{+} d z
\end{aligned}
$$


(see $(4.3))$, so

$$
\left\langle A\left(y_{0}-u_{0}\right),\left(y_{0}-u_{0}\right)^{+}\right\rangle+\int_{\Omega}\left(\beta+\widehat{c}_{1}\right)\left(y_{0}-u_{0}\right)\left(y_{0}-u_{0}\right)^{+} d z=0,
$$

thus

$$
\sigma\left(\left(y_{0}-u_{0}\right)^{+}\right)+\widehat{c}_{1}\left\|\left(y_{0}-u_{0}\right)^{+}\right\|_{2}^{2}=0
$$

and hence

$$
\frac{1}{\widehat{c}_{0}}\left\|\left(y_{0}-u_{0}\right)^{+}\right\|^{2} \leqslant 0
$$

(see (2.14)); hence $y_{0} \leqslant u_{0}$.

Similarly, acting on (4.9) with $\left(v_{0}-y_{0}\right)^{+} \in H_{0}^{1}(\Omega)$, we show that $v_{0} \leqslant y_{0}$. Therefore,

$$
y_{0} \in\left[v_{0}, u_{0}\right]=\left\{u \in H_{0}^{1}(\Omega): v_{0}(z) \leqslant u(z) \leqslant u_{0}(z) \text { for almost all } z \in \Omega\right\},
$$

and so (4.9) becomes

$$
A\left(y_{0}\right)+\beta y_{0}=\lambda N_{g}\left(y_{0}\right)+N_{f}\left(y_{0}\right)
$$

(see (4.3)); thus

$$
y_{0} \in C_{0}^{1}(\bar{\Omega})
$$

(regularity theory; see Struwe [13]), and it solves problem $\left((P)_{\lambda}\right)$.

Moreover, as in the proof of Proposition 3.7, using hypotheses $H_{g}$ (iv) and $H_{f}($ iv), we also show that

$$
u_{0}-y_{0} \in \operatorname{int} C_{+}, \quad y_{0}-v_{0} \in \operatorname{int} C_{+} .
$$

Next we will improve the conclusion of Theorem 4.1 and show that the fifth solution $y_{0}$ is nodal (sign changing). To do this we need to strengthen a little bit the hypotheses on $f(z, \cdot)$.

The new hypotheses of $f$ are the following:

$H_{f}^{\prime}: f: \Omega \times \mathbb{R} \rightarrow \mathbb{R}$ is a Carathéodory function, such that $f(z, 0)=0$ for almost all $z \in \Omega$,

(i), (iii), (iv) are the same as the corresponding hypotheses $H_{f}(\mathrm{i})$, (iii), (iv), 
(ii) we have

$$
\lim _{\zeta \rightarrow \pm \infty} \frac{f(z, \zeta)}{\zeta}=+\infty \quad \text { uniformly for almost all } z \in \Omega
$$

Remark 4.2. Hypothesis $H_{f}^{\prime}$ is a slight restricted version of hypothesis $H_{f}$. Note that if

$$
\widehat{\xi}(z, \zeta)=f(z, \zeta) \zeta-2 F(z, \zeta)
$$

and there exists a function $\widehat{\vartheta}^{*} \in L^{1}(\Omega)_{+}$, such that

$$
\widehat{\xi}(z, \zeta) \leqslant \widehat{\xi}(z, y)+\widehat{\vartheta}^{*}(z) \text { for almost all } z \in \Omega \text {, all } 0 \leqslant \zeta \leqslant y \text { or } y \leqslant \zeta \leqslant 0,
$$

then (4.20) and $H_{f}$ (ii) imply $H_{f}^{\prime}$ (ii) (see Li and Yang [10]). Also, note that hypotheses $H_{f}(\mathrm{i}$ ), (ii), and (iii) imply that there exists $c_{*}>0$, such that

$$
f(z, \zeta) \zeta+c_{*} \zeta^{2} \geqslant 0 \quad \text { for almost all } z \in \Omega \text {, all } \zeta \in \mathbb{R}
$$

We consider the following auxiliary Dirichlet problem:

$$
\begin{gathered}
-\Delta u(z)+\beta(z) u(z)=\lambda c_{1}|u(z)|^{q-2} u(z)-c_{*} u(z) \quad \text { in } \Omega, \\
\left.u\right|_{\partial \Omega}=0 .
\end{gathered}
$$

Here $c_{1}>0$ and $q \in(1,2)$ are as in hypothesis $H_{g}$ (iii) and $c_{*}>0$ is as in $(4.21)$.

Proposition 4.3. For every $\lambda>0$, problem $\left((Q)_{\lambda}\right)$ has a unique nontrivial positive solution $\underline{u}_{\lambda} \in$ int $C_{+}$, and by oddness, we have that $-\underline{u}_{\lambda}=\bar{v}_{\lambda} \in-\operatorname{int} C_{+}$is the unique negative solution of $\left((Q)_{\lambda}\right)$.

Proof. Let $k_{\lambda}^{+}: \Omega \times \mathbb{R} \rightarrow \mathbb{R}$ be the Carathéodory function, defined by

$$
k_{\lambda}^{+}(z, \zeta)= \begin{cases}0 & \text { if } \zeta \leqslant 0 \\ \lambda c_{1} \zeta^{q-1}-\left(\widehat{c}_{1}-c_{*}\right) \zeta & \text { if } \zeta>0\end{cases}
$$

Clearly, we can always assume that $c_{*} \geqslant \widehat{c}_{1}$ (see (4.21)). Let

$$
K_{\lambda}^{+}(z, \zeta)=\int_{0}^{\zeta} k_{\lambda}^{+}(z, s) d s,
$$

and consider the $C^{1}$-functional $\theta_{\lambda}^{+}: H_{0}^{1}(\Omega) \rightarrow \mathbb{R}$, defined by

$$
\theta_{\lambda}^{+}(u)=\frac{1}{2} \sigma(u)+\frac{\widehat{c}_{1}}{2}\|u\|_{2}^{2}-\int_{\Omega} K_{\lambda}^{+}(z, u(z)) d z \quad \forall u \in H_{0}^{1}(\Omega) .
$$


Using (2.14) and (4.22), we have

$$
\theta_{\lambda}^{+}(u) \geqslant \frac{1}{2 \widehat{c}_{0}}\|u\|^{2}+\frac{c_{*}-\widehat{c}_{1}}{2}\left\|u^{+}\right\|_{2}^{2}-\frac{\lambda c_{1}}{q}\left\|u^{+}\right\|_{q}^{q} .
$$

Since $q<2$, from (4.25), we infer that $\theta_{\lambda}^{+}$is coercive. Also, it is sequentially weakly lower semicontinuous (recall that $c_{*} \geqslant \widehat{c}_{1}$ ). So, by the Weierstrass theorem, we can find $\underline{u}_{\lambda} \in H_{0}^{1}(\Omega)$, such that

$$
\theta_{\lambda}^{+}\left(\underline{u}_{\lambda}\right)=\inf _{u \in H_{0}^{1}(\Omega)} \theta_{\lambda}^{+}(u)
$$

As before (see the proof of Proposition 3.7), since $q<2$, we have

$$
\theta_{\lambda}^{+}\left(\underline{u}_{\lambda}\right)<0=\theta_{\lambda}^{+}(0),
$$

hence $\underline{u}_{\lambda} \neq 0$. From (4.26), we have

$$
\left(\theta_{\lambda}^{+}\right)^{\prime}\left(\underline{u}_{\lambda}\right)=0
$$

so

$$
A\left(\underline{u}_{\lambda}\right)+\left(\beta+\widehat{c}_{1}\right) \underline{u}_{\lambda}=N_{k_{\lambda}^{+}}\left(\underline{u}_{\lambda}\right) .
$$

Acting on (4.29) with $\underline{-u}_{\lambda}^{-} \in H_{0}^{1}(\Omega)$, we show that $\underline{u}_{\lambda} \geqslant 0, \underline{u}_{\lambda} \neq 0$ (see (2.14)). Then (4.29) becomes

$$
A\left(\underline{u}_{\lambda}\right)+\left(\beta+c_{*}\right) \underline{u}_{\lambda}=\lambda c_{1} \underline{u}_{\lambda}^{q-1},
$$

so

$$
-\Delta \underline{u}_{\lambda}(z)+\left(\beta(z)+c_{*}\right) \underline{u}_{\lambda}(z) \geqslant 0 \text { for almost all } z \in \Omega,
$$

thus

$$
\Delta \underline{u}_{\lambda}(z) \leqslant\left(\left\|\beta^{+}\right\|_{\infty}+c_{*}\right) \underline{u}_{\lambda}(z) \text { for almost all } z \in \Omega
$$

(see hypothesis $H_{\beta}$ ), and hence

$$
\underline{u}_{\lambda} \in \operatorname{int} C_{+}
$$

(see Vázquez [15] and Pucci and Serrin [14, page 120]). 
We claim that this solution is the unique nontrivial positive solution of $\left((Q)_{\curlywedge}\right)$. To this end, let $u, v \in$ int $C_{+}$be two positive solutions of $\left((Q)_{\lambda}\right)$. We have

$$
\begin{aligned}
\int_{\Omega} \frac{\lambda c_{1} u^{q-1}-c_{*} u}{u}\left(u^{2}-v^{2}\right) d z \\
=\int_{\Omega}\left(\lambda c_{1} u^{q-1}-c_{*} u\right)\left(u-\frac{v^{2}}{u}\right) d z \\
=\int_{\Omega}(-\Delta u)\left(u-\frac{v^{2}}{u}\right) d z+\int_{\Omega} \beta\left(u^{2}-v^{2}\right) d z \\
=\int_{\Omega}\left(\nabla u, \nabla u-\nabla\left(\frac{v^{2}}{u}\right)\right)_{\mathbb{R}^{N}} d z+\int_{\Omega} \beta\left(u^{2}-v^{2}\right) d z \\
=\|\nabla u\|_{2}^{2}-\int_{\Omega}\left(\nabla u, \frac{2 v}{u} \nabla v-\frac{v^{2}}{u^{2}} \nabla u\right)_{\mathbb{R}^{N}} d z+\int_{\Omega} \beta\left(u^{2}-v^{2}\right) d z \\
=\|\nabla u\|_{2}^{2}-\int_{\Omega} \frac{2 v}{u}(\nabla u, \nabla v)_{\mathbb{R}^{N}} d z \\
\quad+\int_{\Omega} \frac{v^{2}}{u^{2}}\|\nabla u\|^{2} d z+\int_{\Omega} \beta\left(u^{2}-v^{2}\right) d z .
\end{aligned}
$$

Interchanging the roles of $u$ and $v$ in the above argument, we also have

$$
\begin{aligned}
& \int_{\Omega} \frac{\lambda c_{1} v^{q-1}-c_{*} v}{v}\left(v^{2}-u^{2}\right) d z \\
& \quad=\|\nabla v\|_{2}^{2}-\int_{\Omega} \frac{2 u}{v}(\nabla v, \nabla u)_{\mathbb{R}^{N}} d z+\int_{\Omega} \frac{u^{2}}{v^{2}}\|\nabla v\|^{2} d z+\int_{\Omega} \beta\left(v^{2}-u^{2}\right) d z
\end{aligned}
$$

Adding (4.34) and (4.35), we obtain

$$
\int_{\Omega}\left(\frac{\lambda c_{1} u^{q-1}-c_{*} u}{u}-\frac{\lambda c_{1} v^{q-1}-c_{*} v}{v}\right)\left(u^{2}-v^{2}\right) d z=\left\|\nabla u-\frac{u}{v} \nabla v\right\|_{2}^{2}+\left\|\nabla v-\frac{v}{u} \nabla u\right\|_{2}^{2} \geqslant 0
$$

Since $q<2$, the function $\zeta \mapsto\left(\lambda_{c_{1}} \zeta^{q-1}-c_{*} \zeta\right) / \zeta$ is strictly decreasing on $(0,+\infty)$. Hence, from (4.36), we infer that $u=v$. This proves the uniqueness of the nontrivial positive solution $\underline{u}_{\lambda} \in$ int $C_{+}$of problem $\left((Q)_{\lambda}\right)$.

The oddness of problem $\left((Q)_{\lambda}\right)$ implies that $\bar{v}_{\lambda}=-\underline{u}_{\lambda} \in-\operatorname{int} C_{+}$is the unique nontrivial negative solution of $\left((Q)_{\curlywedge}\right)$.

Using Proposition 4.3, we can show that problem $\left((P)_{\lambda}\right)$ (for $\left.\lambda \in\left(0, \lambda^{*}\right)\right)$ has extremal constant sign solutions; that is, it has a smallest nontrivial positive solution and a biggest nontrivial negative solution. 
Proposition 4.4. If hypotheses $H_{g}, H_{f^{\prime}}^{\prime} H_{0}$, and $H_{\beta}$ hold and $\lambda \in\left(0, \lambda^{*}\right)$, then problem $\left((P)_{\lambda}\right)$ has a smallest nontrivial positive solution $u_{+}^{\lambda} \in$ int $C_{+}$and a biggest nontrivial negative solution $v_{-}^{\lambda} \in$ int $-C_{+}$.

Proof. Let $\bar{u}$ be a nontrivial positive solution of $\left((P)_{\lambda}\right)$. From the proof of Proposition 3.6, we know that $\bar{u} \in \operatorname{int} C_{+}$. We have

$$
-\Delta \bar{u}(z)+\beta(z) \bar{u}(z)=\lambda g(z, \bar{u}(z))+f(z, \bar{u}(z)) \geqslant \lambda c_{1} \bar{u}(z)^{q-1}-c_{*} \bar{u}(z),
$$

for almost all $z \in \Omega$ (see hypothesis $H_{g}$ (iii) and (4.21)).

We consider the following Carathéodory function:

$$
\widehat{k}_{\lambda}^{+}(z, \zeta)= \begin{cases}0 & \text { if } \zeta \leqslant 0, \\ \lambda c_{1} \zeta^{q-1}+\left(\widehat{c}_{1}-c_{*}\right) \zeta & \text { if } 0 \leqslant \zeta \leqslant \bar{u} \\ \lambda c_{1} \bar{u}(z)^{q-1}+\left(\widehat{c}_{1}-c_{*}\right) \bar{u}(z) & \text { if } \bar{u}<\zeta .\end{cases}
$$

Let

$$
\widehat{K}_{\lambda}^{+}(z, \zeta)=\int_{0}^{\zeta} \widehat{k}_{\lambda}^{+}(z, s) d s,
$$

and consider the $C^{1}$-functional $\xi_{\lambda}^{+}: H_{0}^{1}(\Omega) \rightarrow \mathbb{R}$, defined by

$$
\xi_{\curlywedge}^{+}(u)=\frac{1}{2} \sigma(u)+\frac{\widehat{c}_{1}}{2}\|u\|_{2}^{2}-\int_{\Omega} \widehat{K}_{\lambda}^{+}(z, u(z)) d z \quad \forall u \in H_{0}^{1}(\Omega) .
$$

From (4.38) and (2.14), it is clear that $\xi_{\lambda}^{+}$is coercive. Also $\xi_{\lambda}^{+}$is sequentially weakly lower semicontinuous. Thus we can find $w_{0} \in H_{0}^{1}(\Omega)$, such that

$$
\xi_{\curlywedge}^{+}\left(w_{0}\right)=\inf _{u \in H_{0}^{1}(\Omega)} \xi_{\curlywedge}^{+}(u)
$$

As before, the presence of the "concave" term $\lambda_{c_{1}} \zeta^{q-1}$ implies that

$$
\xi_{\lambda}^{+}\left(w_{0}\right)<0=\xi_{\lambda}^{+}(0)
$$

that is, $w_{0} \neq 0$. From (4.41), we have

$$
\left(\xi_{\curlywedge}^{+}\right)^{\prime}\left(w_{0}\right)=0
$$

so

$$
A\left(w_{0}\right)+\left(\beta+\widehat{c}_{1}\right) w_{0}=N_{\widehat{k}_{\lambda}^{+}}\left(w_{0}\right)
$$


On (4.44) we act with $-w_{0}^{-} \in H_{0}^{1}(\Omega)$ and obtain

$$
\sigma\left(w_{0}^{-}\right)+\widehat{c}_{1}\left\|w_{0}^{-}\right\|_{2}^{2}=0
$$

(see $(4.38))$, so

$$
\frac{1}{\widehat{c}_{0}}\left\|w_{0}^{-}\right\|^{2} \leqslant 0
$$

(see (2.14)), and hence $w_{0} \geqslant 0, w_{0} \neq 0$.

Also, on (4.44) we act with $\left(w_{0}-\bar{u}\right)^{+} \in H_{0}^{1}(\Omega)$. Then

$$
\begin{aligned}
& \left\langle A\left(w_{0}\right),\left(w_{0}-\bar{u}\right)^{+}\right\rangle+\int_{\Omega}\left(\beta+\widehat{c}_{1}\right) w_{0}\left(w_{0}-\bar{u}\right)^{+} d z \\
& \quad=\int_{\Omega} \widehat{k}_{\lambda}^{+}\left(z, w_{0}\right)\left(w_{0}-\bar{u}\right)^{+} d z \\
& \quad=\int_{\Omega}\left(\lambda c_{1} \bar{u}^{q-1}-c_{*} \bar{u}\right)\left(w_{0}-\bar{u}\right)^{+} d z \\
& \quad \leqslant\left\langle A(\bar{u}),\left(w_{0}-\bar{u}\right)^{+}\right\rangle+\int_{\Omega} \beta\left(\left(w_{0}-\bar{u}\right)^{+}\right) d z+\widehat{c}_{1}\left\|\left(w_{0}-\bar{u}\right)^{+}\right\|_{2}^{2}
\end{aligned}
$$

(see (4.38) and (4.37)), so

$$
\sigma\left(\left(w_{0}-\bar{u}\right)^{+}\right)+\widehat{c}_{1}\left\|\left(w_{0}-\bar{u}\right)^{+}\right\|_{2}^{2} \leqslant 0,
$$

thus

$$
\frac{1}{\widehat{c}_{0}}\left\|\left(w_{0}-\bar{u}\right)^{+}\right\|^{2} \leqslant 0
$$

(see (2.14)), and hence

$$
w_{0} \leqslant \bar{u}
$$

So, we have proved that

$$
w_{0} \in[0, \bar{u}]=\left\{u \in H_{0}^{1}(\Omega): 0 \leqslant u(z) \leqslant \bar{u}(z) \text { for almost all } z \in \Omega\right\} .
$$

This means that (4.44) becomes

$$
A\left(w_{0}\right)+\beta w_{0}=\lambda c_{1} w_{0}^{q-1}-c_{*} w_{0}
$$


so $w_{0} \in \operatorname{int} C_{+}$(regularity theory of Struwe [13] and strong maximum principle due to Vázquez [15] and Pucci Serrin [14, page 120]) and it solves problem $\left((Q)_{\lambda}\right)$. Thus

$$
w_{0}=\underline{u}_{\lambda}
$$

(see Proposition 4.3), and

$$
\underline{u}_{\lambda} \leqslant \underline{u}
$$

This shows that every nontrivial positive solution $u$ of $\left((P)_{\curlywedge}\right)$ satisfies

$$
\underline{u}_{\lambda} \leqslant u \text {. }
$$

Similarly, we show that every nontrivial negative solution $v$ of problem $\left((P)_{\lambda}\right)$ satisfies

$$
v \leqslant \bar{v}_{\lambda}=-\underline{u}_{\lambda} .
$$

Let $S_{+}(\lambda)$ (resp., $S_{-}(\lambda)$ ) be the set of nontrivial positive (resp., negative) solutions of problem $\left((P)_{\lambda}\right)$. Let $C \subseteq S_{+}(\lambda)$ be a chain (i.e., a totally ordered subset of $S_{+}(\lambda)$ ). From Dunford and Schwartz [18, page 336], we can find a sequence $\left\{u_{n}\right\}_{n \geqslant 1} \subseteq C$, such that

$$
\inf C=\inf _{n \geqslant 1} u_{n}
$$

Lemma 1.5 of Heikkilä and Lakshmikantham [19, page 15] implies that we can have the sequence $\left\{u_{n}\right\}_{n \geqslant 1} \subseteq C$ to be decreasing. Then we have

$$
A\left(u_{n}\right)+\beta u_{n}=\lambda N_{g}\left(u_{n}\right)+N_{f}\left(u_{n}\right), \quad \underline{u}_{\lambda} \leqslant u_{n} \leqslant u_{1} \forall n \geqslant 1,
$$

so

$$
\text { the sequnece }\left\{u_{n}\right\}_{n \geqslant 1} \subseteq H_{0}^{1}(\Omega) \text { is bounded. }
$$

Hence by passing to a suitable subsequence if necessary, we may assume that

$$
\begin{gathered}
u_{n} \longrightarrow u_{*} \text { weakly in } H_{0}^{1}(\Omega), \\
u_{n} \longrightarrow u_{*} \text { in } L^{2 s^{\prime}}(\Omega) \text { and in } L^{r}(\Omega) .
\end{gathered}
$$

So, passing to the limit as $n \rightarrow+\infty$ in (4.58) and using (4.61), we obtain

$$
A\left(u_{*}\right)+\beta u_{*}=\lambda N_{g}\left(u_{*}\right)+N_{f}\left(u_{*}\right), \quad \underline{u}_{\lambda} \leqslant u_{*}
$$


so

$$
\inf C=u_{*} \in S_{+}(\lambda)
$$

Since $C$ was an arbitrary chain, invoking the Kuratowski-Zorn lemma, we infer that $S_{+}(\lambda)$ has a minimal element $u_{+}^{\lambda} \in \operatorname{int} C_{+}$. From Gasiński and Papageorgiou [17, Lemma 4.2, page 5763], we know that $S_{+}(\lambda)$ is downward directed (i.e., if $u_{1}, u_{2} \in S_{+}(\lambda)$; then we can find $u \in S_{+}(\lambda)$, such that $u \leqslant u_{1}$ and $\left.u \leqslant u_{2}\right)$. So, it follows that $u_{+}^{\lambda}$ is the smallest nontrivial positive solution of problem $\left((P)_{\curlywedge}\right)$.

Similarly, we introduce the biggest nontrivial negative solution $v_{-}^{\lambda} \in-\operatorname{int} C_{+}$of problem $\left((P)_{\lambda}\right)$. Note that $S_{-}(\lambda)$ is upward directed (i.e., if $v_{1}, v_{2} \in S_{-}(\lambda)$, then we can find $v \in S_{-}(\lambda)$, such that $v_{1} \leqslant v$ and $v_{2} \leqslant v$; see Gasiński and Papageorgiou [17, Lemma 4.3, page 5764]).

Now that we have these extremal constant sign solutions, we can produce a nodal solution of problem $\left((P)_{\lambda}\right)$ (with $\lambda \in\left(0, \lambda^{*}\right)$ ).

Theorem 4.5. If hypotheses $H_{g}, H_{f}^{\prime}, H_{0}$, and $H_{\beta}$ hold and $\lambda \in\left(0, \lambda^{*}\right)$, then problem $\left((P)_{\lambda}\right)$ has at least five nontrivial smooth solutions:

$$
\begin{gathered}
u_{0}, \widehat{u} \in \operatorname{int} C_{+}, \quad \widehat{u}-u_{0} \in \operatorname{int} C_{+}, \quad v_{0}, \widehat{v} \in-\operatorname{int} C_{+}, \quad v_{0}-\widehat{v} \in \operatorname{int} C_{+} \\
y_{0} \in C_{0}^{1}(\bar{\Omega}) \text { nodal with } u_{0}-y_{0} \in \operatorname{int} C_{+}, \quad y_{0}-v_{0} \in \operatorname{int} C_{+} .
\end{gathered}
$$

Moreover, problem $\left((P)_{\lambda}\right)$ has a smallest nontrivial positive solution and a biggest negative solution.

Proof. The existence of extremal nontrivial constant sing solutions is guaranteed by Proposition 4.4. Let $u_{+}^{\lambda} \in$ int $C_{+}$and $v_{-}^{\lambda} \in-$ int $C_{+}$be these two extremal solutions.

We introduce the following truncation perturbation of the reaction of problem $\left((P)_{\curlywedge}\right)$ :

$$
r_{\lambda}(z, \zeta)= \begin{cases}\lambda g\left(z, v_{-}^{\lambda}(z)\right)+f\left(z, v_{-}^{\lambda}(z)\right)+\widehat{c}_{1} v_{-}^{\lambda}(z) & \text { if } \zeta<v_{-}^{\lambda}(z) \\ \lambda g(z, \zeta)+f(z, \zeta)+\widehat{c}_{1} \zeta & \text { if } v_{-}^{\lambda}(z) \leqslant \zeta \leqslant u_{+}^{\lambda}(z) \\ \lambda g\left(z, u_{+}^{\lambda}(z)\right)+f\left(z, u_{+}^{\lambda}(z)\right)+\widehat{c}_{1} u_{+}^{\lambda}(z) & \text { if } u_{+}^{\lambda}(z)<\zeta .\end{cases}
$$

This is a Carathéodory function. We set

$$
\Gamma_{\lambda}(z, \zeta)=\int_{0}^{\zeta} r_{\lambda}(z, s) d s
$$

and consider the $C^{1}$-functional $\chi_{\curlywedge}: H_{0}^{1}(\Omega) \rightarrow \mathbb{R}$, defined by

$$
\chi_{\lambda}(u)=\frac{1}{2} \sigma(u)+\frac{\widehat{c}_{1}}{2}\|u\|_{2}^{2}-\int_{\Omega} \Gamma_{\lambda}(z, u(z)) d z \quad \forall u \in H_{0}^{1}(\Omega) .
$$


Also, we introduce

$$
r_{\lambda}^{ \pm}(z, \zeta)=r_{\lambda}\left(z, \pm \zeta^{ \pm}\right), \quad \Gamma_{\lambda}^{ \pm}(z, \zeta)=\int_{0}^{\zeta} r_{\lambda}^{ \pm}(z, s) d s
$$

and consider the $C^{1}$-functionals $\chi_{\lambda}^{ \pm}: H_{0}^{1}(\Omega) \rightarrow \mathbb{R}$, defined by

$$
\chi_{\lambda}^{ \pm}(u)=\frac{1}{2} \sigma(u)+\frac{\widehat{c}_{1}}{2}\|u\|_{2}^{2}-\int_{\Omega} \Gamma_{\lambda}^{ \pm}(z, u(z)) d z \quad \forall u \in H_{0}^{1}(\Omega) .
$$

As in the proof of Theorem 4.1, we show that

$$
K_{x_{\lambda}} \subseteq\left[v_{-}^{\lambda}, u_{+}^{\lambda}\right], \quad K_{x_{\lambda}^{+}} \subseteq\left[0, u_{+}^{\lambda}\right], \quad K_{x_{\lambda}^{-}} \subseteq\left[v_{-}^{\lambda}, 0\right]
$$

(see (4.65)). The extremality of the solutions $v_{-}^{\curlywedge}$ and $u_{+}^{\curlywedge}$ implies that

$$
K_{x_{\lambda}} \subseteq\left[v_{-}^{\curlywedge}, u_{+}^{\lambda}\right], \quad K_{x_{\lambda}^{+}}=\left\{0, u_{+}^{\curlywedge}\right\}, \quad K_{x_{\lambda}^{-}}=\left\{v_{-}^{\curlywedge}, 0\right\} .
$$

Claim 4. Solutions $u_{+}^{\lambda}$ and $v_{-}^{\curlywedge}$ are both local minimizers of $\chi_{\lambda}$.

Evidently $X_{\lambda}^{+}$is coercive (see (4.65)) and sequentially weakly lower semicontinuous. So, we can find $u_{+} \in H_{0}^{1}(\Omega)$, such that

$$
X_{\lambda}^{+}\left(u_{+}\right)=\inf _{u \in H_{0}^{1}(\Omega)} X_{\lambda}^{+}(u)
$$

As before (see the proof of Proposition 3.7), the presence of the "concave" term implies that

$$
x_{\lambda}^{+}\left(u_{+}\right)<0=x_{\lambda}^{+}(0)
$$

hence $u_{+} \neq 0$, and so $u_{+}=u_{+}^{\curlywedge}$ (see (4.71)). Since

$$
\left.x_{\lambda}\right|_{C_{+}}=\left.x_{\lambda}^{+}\right|_{C_{+}}
$$

it follows that $u_{+}=u_{+}^{\lambda} \in$ int $C_{+}$is local $C_{0}^{1}(\bar{\Omega})$-minimizers of $\chi_{\lambda}$; hence by Brézis and Nirenberg [16], it is also local $H_{0}^{1}(\Omega)$-minimizers of $\chi_{\lambda}$.

Similarly this is for $v_{-}^{\lambda}$ using this time the functional $\chi_{\lambda}^{-}$. This proves the claim.

Without any loss of generality, we may assume that

$$
x_{\lambda}\left(v_{-}^{\curlywedge}\right) \leqslant x_{\lambda}\left(u_{+}^{\lambda}\right)
$$


The analysis is similar, if the opposite inequality holds. Because of the claim, we can find $\varrho \in(0,1)$ small, such that

$$
x_{\lambda}\left(v_{-}^{\lambda}\right) \leqslant x_{\lambda}\left(u_{+}^{\lambda}\right)<\inf \left\{x_{\lambda}(u):\left\|u-u_{+}^{\lambda}\right\|=\rho\right\}=\eta_{\lambda}
$$

(see Gasiński and Papageorgiou [17, proof of Theorem 3.4]).

Since $\chi_{\lambda}$ is coercive (see (4.65)), it satisfies the Cerami condition. This fact and (4.76) permit the use of the mountain pass theorem (see Theorem 2.1). So, we can find $y_{0} \in K_{x_{\imath}} \subseteq$ $\left[v_{-}^{\curlywedge}, u_{+}^{\curlywedge}\right]$ (see $\left.(4.71)\right)$, such that

$$
\eta_{\lambda} \leqslant \chi_{\lambda}\left(y_{0}\right)
$$

so

$$
y_{0} \notin\left\{v_{-}^{\curlywedge}, u_{+}^{\curlywedge}\right\}
$$

(see (4.76)).

Since $y_{0}$ is a critical point of $\chi_{\lambda}$ of mountain pass type, we have

$$
C_{1}\left(x_{\curlywedge}, y_{0}\right) \neq 0
$$

(see e.g., Chang [20]). On the other hand, hypothesis $H_{f}$ (iii) implies that we can find $\widehat{\xi}_{1}>0$, such that

$$
f(z, \zeta) \zeta-\mu F(z, \zeta) \leqslant \widehat{\xi}_{1} \zeta^{2} \text { for almost all } z \in \Omega, \text { all }|\zeta| \leqslant \delta_{1}
$$

for some $\delta_{1} \leqslant \delta_{0}$. This combined with hypothesis $H_{g}$ (iii) implies that

$$
\begin{aligned}
\mu \lambda G(z, \zeta)+\mu F(z, \zeta) & \geqslant \lambda g(z, \zeta) \zeta+f(z, \zeta) \zeta \\
& >0 \text { for almost all } z \in \Omega, \text { all }|\zeta| \leqslant \delta_{2}
\end{aligned}
$$

for some $\delta_{2} \leqslant \delta_{1}$ and

$$
\text { ess } \sup \lambda G\left(\cdot, \delta_{2}\right)+F\left(\cdot, \delta_{2}\right)>0 \text {. }
$$

Hence invoking Proposition 2.1 of Jiu and Su [21], we infer that

$$
C_{k}\left(\chi_{\lambda}, 0\right)=0 \quad \forall k \geqslant 0
$$

Combining (4.79) and (4.83), we have that $y_{0} \neq 0$. Since $y_{0} \in\left[v_{-}^{\lambda}, u_{+}^{\lambda}\right]$, the extremality of $v_{-}^{\lambda}$ and $u_{+}^{\lambda}$ implies that $y_{0}$ must be a nodal solution of problem $\left((P)_{\lambda}\right)$, and the regularity theory (see Struwe [13]) implies that $y_{0} \in C_{0}^{1}(\bar{\Omega})$. 


\section{Acknowledgments}

This research has been partially supported by the Ministry of Science and Higher Education of Poland under Grants no. N201 542438 and N201 604640.

\section{References}

[1] A. Ambrosetti, H. Brezis, and G. Cerami, "Combined effects of concave and convex nonlinearities in some elliptic problems," Journal of Functional Analysis, vol. 122, no. 2, pp. 519-543, 1994.

[2] Y. I'lyasov, "On nonlocal existence results for elliptic equations with convex-concave nonlinearities," Nonlinear Analysis: Theory, Methods \& Applications A, vol. 61, no. 1-2, pp. 211-236, 2005.

[3] S. Li, S. Wu, and H.-S. Zhou, "Solutions to semilinear elliptic problems with combined nonlinearities," Journal of Differential Equations, vol. 185, no. 1, pp. 200-224, 2002.

[4] V. F. Lubyshev, "Multiple solutions of an even-order nonlinear problem with convex-concave nonlinearity," Nonlinear Analysis: Theory, Methods E Applications A, vol. 74, no. 4, pp. 1345-1354, 2011.

[5] V. D. Rădulescu and D. Repovš, "Combined effects in nonlinear problems arising in the study of anisotropic continuous media," Nonlinear Analysis: Theory, Methods E Applications, vol. 75, no. 3, pp. 1524-1530, 2012.

[6] D. Motreanu, V. V. Motreanu, and N. S. Papageorgiou, "On $p$-Laplace equations with concave terms and asymmetric perturbations," Proceedings of the Royal Society of Edinburgh A, vol. 141, no. 1, pp. 171-192, 2011.

[7] L. Gasiński and N. S. Papageorgiou, Nonlinear Analysis, vol. 9 of Series in Mathematical Analysis and Applications, Chapman \& Hall/CRC Press, Boca Raton, Fla, USA, 2006.

[8] D. Motreanu and V. D. Rădulescu, Variational and Non-Variational Methods in Nonlinear Analysis and Boundary Value Problems, vol. 67 of Nonconvex Optimization and Its Applications, Kluwer, Dordrecht, The Netherlands, 2003.

[9] L. Gasiński and N. S. Papageorgiou, "Dirichlet problems with double resonance and an indefinite potential," Nonlinear Analysis: Theory, Methods E Applications, vol. 75, no. 12, pp. 4560-4595, 2012.

[10] G. Li and C. Yang, "The existence of a nontrivial solution to a nonlinear elliptic boundary value problem of $p$-Laplacian type without the Ambrosetti-Rabinowitz condition," Nonlinear Analysis: Theory, Methods \& Applications A, vol. 72, no. 12, pp. 4602-4613, 2010.

[11] L. Gasiński and N. S. Papageorgiou, "Existence and multiplicity of solutions for Neumann $p$ Laplacian-type equations," Advanced Nonlinear Studies, vol. 8, no. 4, pp. 843-870, 2008.

[12] L. Gasiński and N. S. Papageorgiou, Nonsmooth Critical Point Theory and Nonlinear Boundary Value Problems, vol. 8 of Series in Mathematical Analysis and Applications, Chapman \& Hall/CRC Press, Boca Raton, Fla, USA, 2005.

[13] M. Struwe, Variational Methods: Applications to Nonlinear Partial Differential Equations and Hamiltonian Systems, Springer, Berlin, Germany, 1990.

[14] P. Pucci and J. Serrin, The Maximum Principle, vol. 73 of Progress in Nonlinear Differential Equations and their Applications, Birkhäuser, Basle, Switzerland, 2007.

[15] J. L. Vázquez, “A strong maximum principle for some quasilinear elliptic equations," Applied Mathematics and Optimization, vol. 12, no. 3, pp. 191-202, 1984.

[16] H. Brezis and L. Nirenberg, " $H^{1}$ versus $C^{1}$ local minimizers," Comptes Rendus de l'Académie des Sciences. Série I. Mathématique, vol. 317, no. 5, pp. 465-472, 1993.

[17] L. Gasiński and N. S. Papageorgiou, "Nodal and multiple constant sign solutions for resonant $p$ Laplacian equations with a nonsmooth potential," Nonlinear Analysis: Theory, Methods E Applications A, vol. 71, no. 11, pp. 5747-5772, 2009.

[18] N. Dunford and J. T. Schwartz, Linear Operators. I. General Theory, vol. 7 of Pure and Applied Mathematics, Wiley, New York, NY, USA, 1958.

[19] S. Heikkilä and V. Lakshmikantham, Monotone Iterative Techniques for Discontinuous Nonlinear Differential Equations, vol. 181 of Monographs and Textbooks in Pure and Applied Mathematics, Marcel Dekker, New York, NY, USA, 1994.

[20] K.-C. Chang, Infinite-Dimensional Morse Theory and Multiple Solution Problems, vol. 6 of Progress in Nonlinear Differential Equations and their Applications, Birkhäuser Boston, Boston, Mass, USA, 1993.

[21] Q.-S. Jiu and J.-B. Su, "Existence and multiplicity results for Dirichlet problems with $p$-Laplacian," Journal of Mathematical Analysis and Applications, vol. 281, no. 2, pp. 587-601, 2003. 


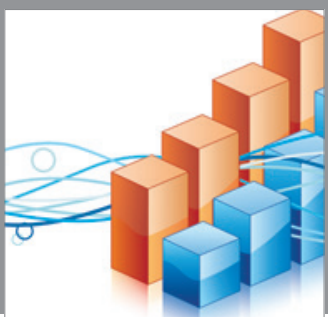

Advances in

Operations Research

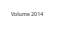

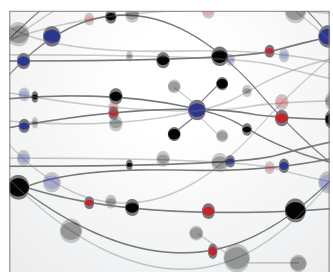

\section{The Scientific} World Journal
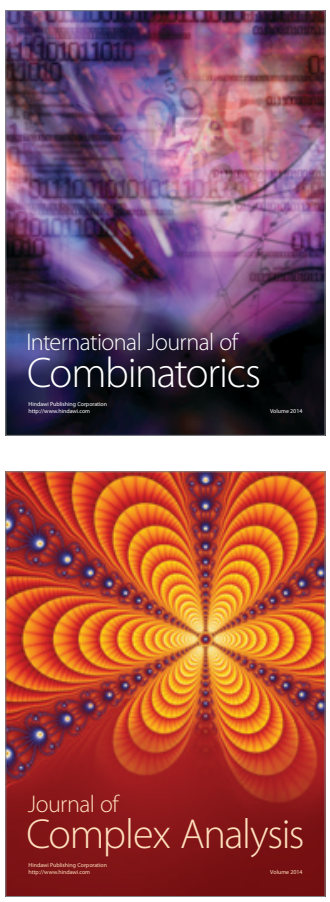

International Journal of

Mathematics and

Mathematical

Sciences
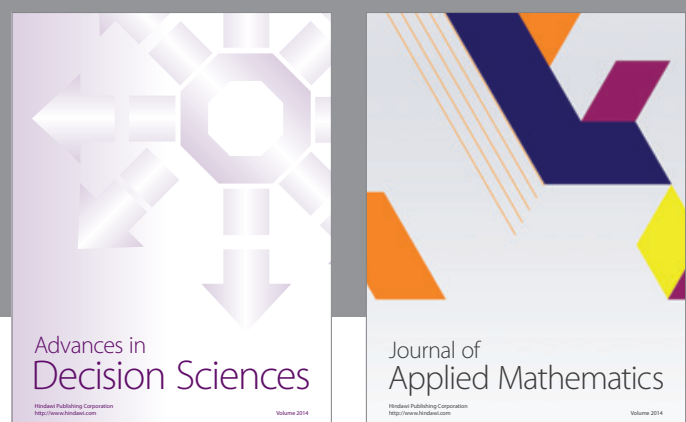

Journal of

Applied Mathematics
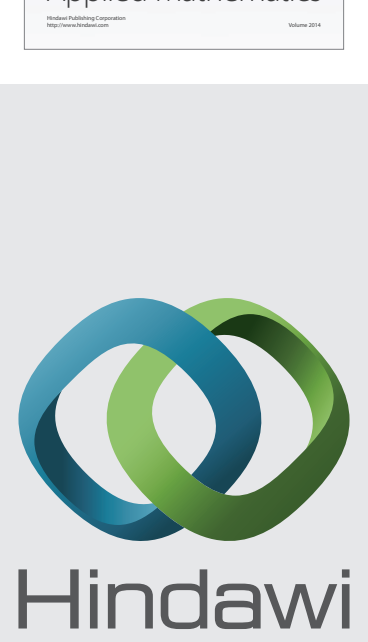

Submit your manuscripts at http://www.hindawi.com
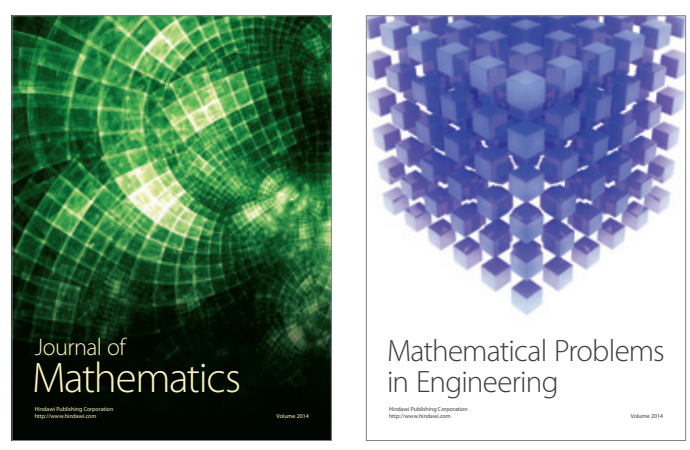

Mathematical Problems in Engineering
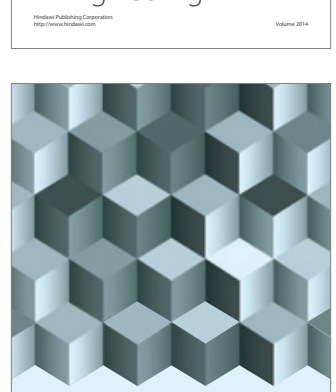

Journal of

Function Spaces
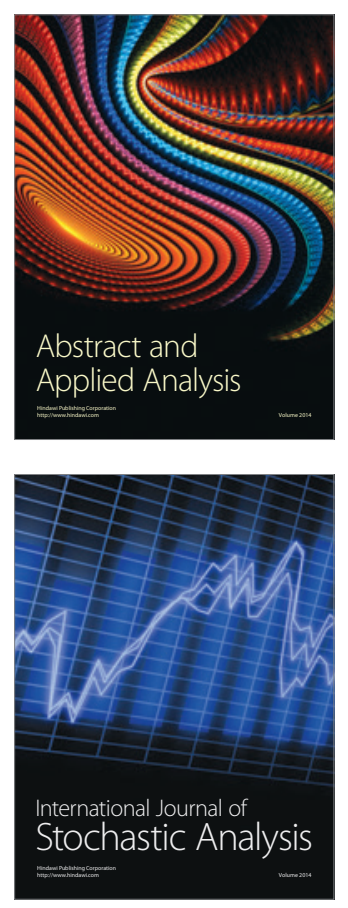

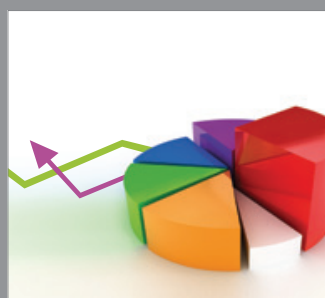

ournal of

Probability and Statistics

Promensencen
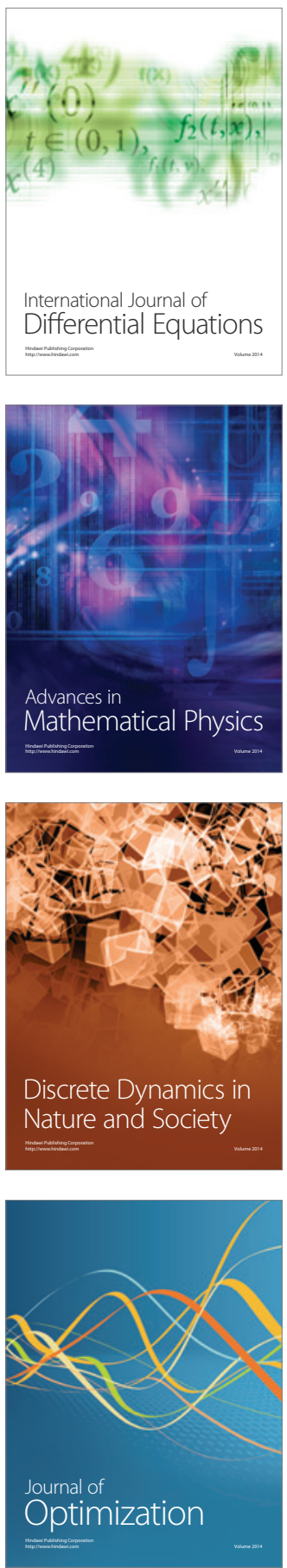